\title{
Security of Underwater and Air-Water Wireless Communication
}

This paper was downloaded from TechRxiv (https://www.techrxiv.org).

\section{LICENSE}

CC BY-NC-SA 4.0

SUBMISSION DATE / POSTED DATE

26-02-2022 / 03-03-2022

\section{CITATION}

Aman, Waqas; Al-Kuwari, Saif; Kumar, Ambrish; Rahman, Muhammad Mahboob ur (2022): Security of Underwater and Air-Water Wireless Communication. TechRxiv. Preprint. https://doi.org/10.36227/techrxiv.19242624.v1

DOI 


\title{
Security of Underwater and Air-Water Wireless Communication
}

\author{
Waqas Aman*, Saif Al-Kuwari*, Ambrish Kumar ${ }^{\dagger}$, and Muhammad Mahboob Ur Rahman* \\ ${ }^{*}$ Division of Information and Computing Technology, College of Science and Engineering, \\ Hamad Bin Khalifa University, Qatar Foundation, Doha, Qatar. \\ ${ }^{\dagger}$ Department of Electronics and Communication Engineering Netaji Subhas University of Technology, New Delhi, India. \\ †Electrical Engineering Department, Information Technology University, Lahore 54000, Pakistan. \\ *waqasaman87@gmail.com, * smalkuwari@hbku.edu.qa, †ambrish.nsit@gmail.com, ${ }^{\star}$ mahboob.rahman@itu.edu.pk
}

\begin{abstract}
We present a first detailed survey that focuses on the security challenges faced by the underwater and air-water (A-W) wireless communication networks (WCNs), as well as the countermeasures proposed to date. Specifically, we provide a detailed literature review of the various kinds of active and passive attacks which hamper the confidentiality, integrity, authentication and availability of both underwater and A-W WCNs. For clarity of exposition, this survey paper is mainly divided into two parts. The first part of the paper is essentially a primer on underwater and A-W WCNs whereby we outline the benefits and drawbacks of the three promising underwater and $A-W$ candidate technologies: radio frequency $(\mathbf{R F})$, acoustic, and optical, along with channel modelling. To this end, we also describe the indirect (relay-aided) and direct mechanisms for the A-W WCNs along with channel modelling. This sets the stage for the second part (and main contribution) of the paper whereby we provide a thorough comparative discussion of a vast set of works that have reported the security breaches (as well as viable countermeasures) for many diverse configurations of the underwater and A-W WCNs. Finally, we highlight some research gaps in the open literature and identify some open problems for the future work.

Index Terms-Underwater wireless communication, air-water communication, wireless communication, acoustic, RF, optics, free-space optics (FSO), underwater acoustic sensor networks (UWASN), direct communication, indirect communication, security, authentication, confidentiality, integrity, availability cryptography, physical layer security, eavesdropping, jamming, impersonation.
\end{abstract}

\section{INTRODUCTION}

Water covers $71 \%$ of the earth's surface (of which $96 \%$ is held by oceans). Many activities, such as hunting for natural resources, trading, military operations, monitoring marine pollution, make the ocean a cardinal spot. Consequently, establishing communication between ground stations, airborne nodes (i.e., satellites, aeroplanes and unmanned aerial vehicles) and underwater sensor nodes, such as submarine and Autonomous Underwater Vehicles (AUVs), are crucial to facilitate previously mentioned activities. Realizing its importance, over the last few decades, researchers have done a plethora of research in this area [1-4].

Over the years, Radio Frequency (RF), acoustic, and optical underwater wireless communication networks (WCNs) have gained widespread acceptance and adoption by the naval and oceanic communities. RF was used in early days of underwater wireless communication but lost its efficacy due to severe attenuation it suffered from, which limited the effective range of communication. Long-range underwater wireless communication was also achieved using RF but with very large antennas [5]. Generally, acoustic underwater WCNs are preferred when long communication range is needed [6], but it is affected by multipath propagation, and the low speed of acoustic waves drastically reduces its rate. At the same time, underwater wireless optical communication is becoming popular due to its high rate communication, but with moderate communication range [1]. On the other hand, AirWater (A-W) wireless communication where some airborne nodes communicate with underwater nodes either directly or through relay/surface node has recently got the attention of the research community. RF and optics are preferred and are commonly used on the over water part of A-W wireless communication $[7,8]$.

Wireless communication provides portability and mobility to the nodes by taking advantage of their shared mediums (i.e., air, and water). However, the broadcast nature of wireless communication (i.e., underwater and (A-W)) makes it vulnerable to many types of malicious attacks, such as eavesdropping, spoofing, denial of service, and jamming. Such attacks/vulnerabilities can disrupt the confidentiality, integrity, authentication and availability of information traversing networks. In fact, vulnerabilities in communication systems used for underwater and $\mathrm{A}-\mathrm{W}$ wireless communication have already been demonstrated [9-11]. Therefore, in order to preserve security of WCNs, researchers investigated the various attacks on the networks and proposed various counter-mechanisms.

In this survey, we focus on the security aspects of underwater and A-W wireless communication networks. Specifically, we review the works on the four fundamental properties of security (i.e., confidentiality, integrity, authentication and availability) in underwater and $\mathrm{A}-\mathrm{W}$ wireless communication systems. 


\begin{tabular}{|c|c|c|c|c|c|}
\hline Survey & \multicolumn{2}{|c|}{ Security of Underwater Wireless Communication } & \multicolumn{2}{c|}{$\begin{array}{c}\text { Security of Air-Water Wireless Communication } \\
\text { Indirect }\end{array}$} \\
& RF & Acoustic & Optical & $\times$ & $\times$ \\
\hline$[11](2011)$ & $\times$ & $\checkmark$ & $\times$ & $\times$ & $\times$ \\
\hline$[10](2015)$ & $\times$ & $\checkmark$ & $\times$ & $\times$ & $\times$ \\
\hline$[17](2016)$ & $\times$ & $\checkmark$ & $\times$ & $\times$ & $\times$ \\
\hline$[19](2018)$ & $\times$ & $\checkmark$ & $\times$ & $\times$ & $\times$ \\
\hline$[18](2019)$ & $\times$ & $\checkmark$ & $\times$ & $\checkmark$ & $\checkmark$ \\
\hline This Paper & $\checkmark$ & $\checkmark$ & $\checkmark$ & & \\
\hline
\end{tabular}

TABLE I: Our survey paper is more comprehensive than the earlier survey papers as it covers various technologies and various scenarios to address security challenges faced by both underwater and A-W WCNs.

\section{A. Contributions}

The novelty of this survey is as follows:

- Some existing survey papers such as [1-3, 12-16] mainly focus on various communication aspect of the underwater WSNs (e.g., energy efficiency, routing, etc.) Contrary to those works, this paper emphasizes on the security aspect of underwater and A-W WCNs, under diverse configurations and system models.

- Though some existing survey papers discussed security aspects of underwater WCNs, e.g., [10, 11, 17-19]; this paper is the first that focuses on the security aspect of both underwater and A-W WCNs. Additionally, we provide a thorough and updated review of the state-ofthe-art in the field.

- The previous survey papers discuss the underwater WCNs in one medium (i.e., water), and mostly consider acoustic communication technology. On the other hand, we review multiple technologies (i.e., acoustic, RF, optical) and scenarios (i.e., underwater WCNs, and A-W WCNs).

For clarity of exposition, Table I provides a crisp comparison between the earlier survey papers and our paper.

\section{B. Organization.}

The rest of this paper is organized as follows. Section-II introduces underwater wireless communication with a brief discussion on three widely used technologies: RF, acoustic and optical communication. Section-III discusses air-water wireless communication and describes both indirect and the newly developed domain of direct air-water wireless communication. Section-IV reports on the security and state-of-the-art of underwater and air-water wireless communication, focusing on authentication, confidentiality, and integrity. Finally, in Section- $\mathrm{V}$, we conclude the paper and discuss open problems for future work.

\section{UNDERWATER WIRELESS COMMUNICATION}

In underwater wireless communication, submarines, AUVs, and other sensor nodes are typically considered underwater nodes which are wirelessly connected with the surface nodes (e.g., ships, buoys) or airborne nodes (e.g., Airplanes, satellites, drones, unmanned aerial vehicles), as illustrated in Fig. 1.

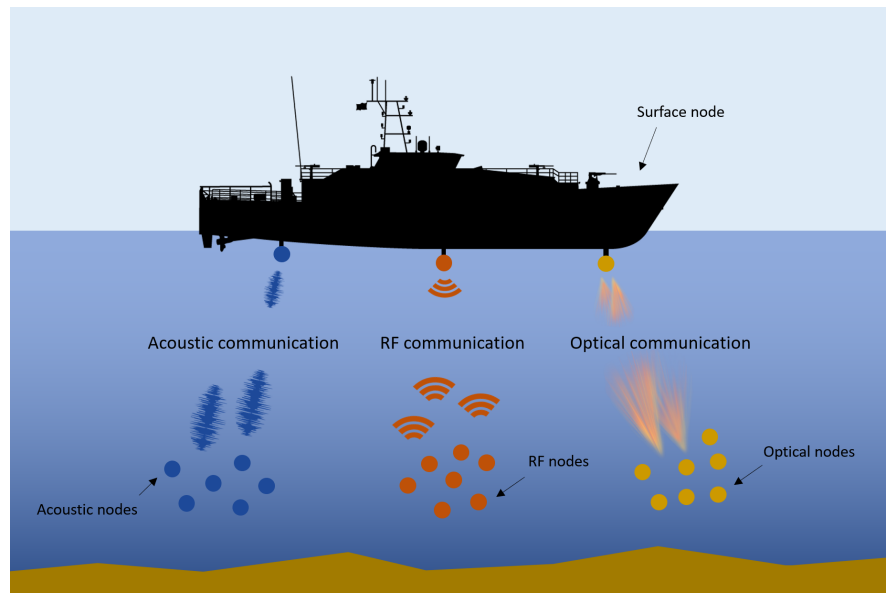

Fig. 1: Illustration of underwater WCNs.

In the following subsections, we discuss the three most widely used technologies for underwater WCNs, namely: RF, acoustic and optical. Again, for clarity of exposition, a compact/crisp comparison of the three technologies is given in Table II.

\section{A. RF-based underwater wireless communication networks}

RF-based underwater WCNs offer many distinct advantages over their acoustic-based and optical-based counterparts; consequently, they have been used for various underwater applications [23]. In fact, RF-based underwater WCNs have a rich history that goes back to early years of $19^{\text {th }}$ century [24]. One prominent example of an legacy RF-based underwater WCN is the so-called Extreme Low Frequency (ELF) communication system deployed by the US NAVY in Wisconsin state with antenna's length exceeding $25 \mathrm{~km}$ [5]. This system enabled one-way communication with the submarines in order to (localize them, and thus) help them reach the surface of water safely. Indeed, due to its Point-toMultipoint (P2MP) propagation nature, RF communication is suitable for cross-medium communication (air-water or waterto-air) where the terrestrial RF-based WCNs connect with the RF-based underwater WCNs [25]. In contrast to their acoustic and optical counterparts, RF-based underwater WCNs are more tolerant to underwater turbulence and turbidity effects [26]. This feature is certainly beneficial when underwater environmental conditions are random and/or unpredictable. 


\begin{tabular}{|c|c|c|c|c|c|c|}
\hline System & Advantages & Disadvantages & $\begin{array}{l}\text { Operating } \\
\text { Frequency } \\
\text { Range (Hz) }\end{array}$ & $\begin{array}{l}\text { Bandwidth } \\
\text { (Hz) }\end{array}$ & Data rates & $\begin{array}{l}\text { Deployment } \\
\text { Example }\end{array}$ \\
\hline $\mathrm{RF}$ & $\begin{array}{llr}\text { Fast carrier } & \text { (velocity } & \approx \\
225,000 \mathrm{~km} / \mathrm{s}), & \text { immune } & \text { to } \\
\text { acoustic noise, } & & \end{array}$ & $\begin{array}{l}\text { High attenuation, large } \\
\text { transceivers, moderate latency, } \\
\text { short range (less than } 10 \mathrm{~m})\end{array}$ & $\begin{array}{l}30-300 \text { for } \\
\text { ELF, MHz for } \\
\text { others }\end{array}$ & $\begin{array}{l}\approx 300 \text { for ELF, } \\
\mathrm{MHz} \text { for others }\end{array}$ & $\begin{array}{l}1-2 \mathrm{Mbps} \text { for } 1- \\
2 \mathrm{~m}, 50-100 \mathrm{bps} \\
\text { for } 200 \mathrm{~m}\end{array}$ & ELF [5] \\
\hline Acoustic & $\begin{array}{l}\text { Travels over long distances (in } \\
\text { kilo meters), small transceivers } \\
\text { (compared to RF) }\end{array}$ & $\begin{array}{l}\text { Low data rates, low bandwidth, } \\
\text { high noises, high latency, bulky } \\
\text { transceivers (compared to opti- } \\
\text { cal) }\end{array}$ & $10-10^{5}$ & $\approx 10 \mathrm{~K}$ & $\begin{array}{l}1.5-50 \mathrm{Kbps} \\
\text { for } 0.5 \mathrm{Km} \text {, } \\
0.6-3 \mathrm{Kbps} \text { for } \\
28-120 \mathrm{Km}\end{array}$ & $\begin{array}{l}\text { Gertrude } \\
{[6], \quad \text { Popoto }} \\
\text { acoustic } \\
\text { modems [20], } \\
\text { Aqua-scent } \\
\text { modems [21] }\end{array}$ \\
\hline Optical & $\begin{array}{l}\text { Fast carrier } \text { (velocity } \approx \\
225,000 \mathrm{~km} / \mathrm{s}, \quad \text { unlicensed } \\
\text { spectrum, high bandwidth, } \\
\text { secure, low latency, light } \\
\text { transceivers }\end{array}$ & $\begin{array}{l}\text { Needs line of sight due to high } \\
\text { directivity of beam, moder- } \\
\text { ate attenuation, medium range } \\
(100 \mathrm{~m})\end{array}$ & $10^{12}-10^{14}$ & $10-150 \mathrm{M}$ & $\begin{array}{l}1 \text { Gbps for } 2 \mathrm{~m}, \\
1 \quad \text { Mbps for } \\
25 \mathrm{~m}\end{array}$ & $\begin{array}{l}\text { LUMA by by } \\
\text { Hydormea [22] }\end{array}$ \\
\hline
\end{tabular}

TABLE II: Comparison of different underwater communication technologies

Furthermore, RF signals can also propagate in adverse water conditions, while the optical waves are susceptible to particles and marine fouling [27]. Unlike acoustic communication, RF radiation is immune to acoustic noise, and it has no known effects on marine life [3].

Despite the advantages RF-based underwater WCNs can offer, the heavy absorption loss at high frequencies and the requirement of excessively large antennas at low frequencies make this technology less attractive for deployment in defense and civilian marine underwater WCNs. Moreover, it also suffers from many other drawbacks, such as high attenuation (beside absorption and scattering, seawater conductivity seriously affects the propagation of electromagnetic waves), low bandwidth, very short communication range (typically less than 10m) and requiring large antennas. Nevertheless, occasionally, RF-based underwater WCNs have been practically deployed, as reported in the literature: [26, 28-36].

\section{RF Channel Modelling}

Attenuation: The main factor of attenuation in the RF underwater channel is the absorption loss. The coefficient of absorption for seawater is given as:

$$
\alpha_{s w}=\sqrt{\pi f \mu \sigma},
$$

where $\sigma$ is the conductivity, $\mu$ is the permeability, and $f$ is the operating frequency. The absorption coefficient for fresh water is given as:

$$
\alpha_{f w}=\frac{\sigma}{2} \sqrt{\frac{\mu}{\epsilon}}
$$

where $\epsilon$ is the permittivity.

Transfer Function: The transfer function of RF is given as [37]:

$$
H(f)=H_{0} e^{-\alpha(f) d} e^{-j \psi(f)},
$$

where $H_{0}$ is a DC channel gain, $d$ is distance, $f$ is the operating frequency and $\psi(f)$ is the phase.
B. Acoustic-based underwater wireless communication networks

Acoustic-based underwater WCNs utilize acoustic modems (that consists of a sensitive acoustic receiver, i.e., hydrophone and an acoustic transmitter) both at the surface node and at the underwater node [38], [39]. The current state-of-the-art in the design of acoustic modems with compact form-factor makes acoustic-based underwater WCNs appealing for many shallow water and deep water applications [40]. Furthermore, the inherent ability of acoustic-based underwater WCNs to provide long-range transmission contributed to its widespread adoption for a plethora of marine applications, such as sound navigation and ranging (SONAR) protocols [41]. However, while the attenuation of acoustic waves at higher frequencies is still severe, the compact form-factor of the acoustic modems remains the preferred advantage, to the oceanic engineers.

Although acoustic-based underwater WCNs have gained widespread adoption, they have certain intrinsic technical limitations as well [42]. For example, the underwater acoustic frequencies vary between $10 \mathrm{~Hz}$ to $1 \mathrm{MHz}$, which results in low transmission rates. Furthermore, the acoustic link is significantly more affected by the water temperature, path loss, noise, multi-path effect, and Doppler spread that might create a severe communication delay from the acoustic source to the acoustic receiver [40]. As a result, acoustic-based underwater WCNs cannot support the applications where real-time large volume data exchange is required. Moreover, their transceivers are bulky, costly, and energy-consuming and can also affect marine life [43].

\section{Acoustic Channel Modelling}

Pathloss: The path-loss of LoS UWA channel between a node pair having distance $d$ separation is given as [44]

$$
P L(d, f)=d^{\nu} \alpha(f)^{d} .
$$

Alternatively, the $\mathrm{dB}$ scale representation of (4) is

$$
P L(d, f)_{\mathrm{dB}}=\nu 10 \log d+d \alpha(f)_{\mathrm{dB}} .
$$


Basically, the path-loss of LoS UWA channel is the summation of spreading $\operatorname{loss}(\nu 10 \log d)$ and absorption loss $\left(d \alpha(f)_{\mathrm{dB}}\right)$ in $\mathrm{dB}$ scale, where $\nu$ is the spreading factor which takes 0 or 1 values, while the absorption coefficient $\alpha(f)_{\mathrm{dB}}$ is given as [44]

$$
\alpha(f)_{\mathrm{dB}}=\frac{0.11 f^{2}}{1+f^{2}}+\frac{44 f^{2}}{4100+f^{2}}+2.75 \times 10^{-4} f^{2}+0.003 .
$$

Noise: There are mainly four sources of noise in the UWA channel: thermal noise, wave noise, shipping noise, and turbulence noise. We discuss these in more detail below:

- Thermal noise: Thermal noise is due to thermal agitation of molecules and operates above the $100 \mathrm{kHz}$ (Note that the noise effect is zero outside the given range of frequencies), and its power spectral density (PSD) in $\mathrm{dB}$ is given as [45]

$$
N_{\mathrm{T}}(f)=-15-20 \log (f) .
$$

- Wave noise: Wave noise is the most prominent component among the four mentioned noises because it exists in the desired frequency range (i.e., $100 \mathrm{~Hz}$ to $100 \mathrm{kHz}$ ). This produces due to the surface movement of the sea, which is induced by the wind. The PSD of wave noise in $\mathrm{dB}$ is given as

$$
N_{\mathrm{W}}(f)=50+20 \log (f)-40 \log (f+0.4)+7.5 \sqrt{w},
$$

where $w$ is the speed of the wind.

- Shipping noise: The noise generated due to shipping activities is shipping noise. This noise exists in the range $10<f<100 \mathrm{~Hz}$. The PSD of shipping noise is given as

$$
N_{\mathrm{S}}(f)=40-60 \log (f+0.3)+26 \log (f)+20(s-0.5),
$$

where $s$ represents the shipping activities (i.e. $s=0(1)$ for low(high)).

- Turbulence noise: Turbulence noise is generated due to random movement of sea surface due to wave currents, and it exists at very low frequencies, i.e., $f<10 \mathrm{~Hz}$. The PSD of Turbulence noise is given as

$$
N_{\operatorname{Tr}}(f)=17-30 \log (f) \text {. }
$$

- Net noise: The PSD of net noise can be expressed as

$$
N_{\mathrm{NN}}(f)=N_{\mathrm{T}}(f)+N_{\mathrm{W}}(f)+N_{\mathrm{S}}(f)+N_{\mathrm{Tr}}(f) .
$$

There is a very good approximate of PSD of net noise in the range $1<f<100 \mathrm{KHz}$ and is given as [44]

$$
N(f)_{\mathrm{dB}} \approx N_{1}-\tau 10 \log (f),
$$

where $N_{1}$ and $\tau$ are the experimental constants.

Multipath: Now, let $H_{j}$ be the channel gain of UWA channel for $j^{t h}$ sub-carrier. Then during $k^{t h}$ block/time-slot, $H_{j}(k)$ which comprises total $L$ paths can be expressed as

$$
H_{j}(k)=\sum_{l=1}^{L} \frac{1}{\sqrt{P L\left(d_{l}, f_{j}\right)}} h_{l}(k) e^{-j 2 \pi f_{j} \xi_{l}(k)},
$$

where $P L\left(d_{l}, f_{j}\right)$ is the path-loss of the $l^{t h}$ path having distance $d_{l}$, and the delay of $l^{t h}$ path is $\xi_{l}(k)=\frac{d_{l}}{v}$, where $v$ is the underwater speed of acoustic wave. $h_{l}(k)$ is the $l^{t h}$ path gain which is modelled as independent, first-order autoregressive process.

\section{Optical-based underwater wireless communication net- works}

Optical-based underwater WCNs utilize (visible or invisible) light to carry information whereby a laser diode (LD) and/or a light emitting diode (LED) are used as the sources for transmission [46-49]. The optical underwater WCNs recently received attention due to their favorable characteristics such as high speed, high data rates, and high bandwidth [47-55]. The effective communication range of optical-based underwater WCN is greater than its RF-based counterpart but quite lower than acoustic-based counterpart (typically, up to $100 \mathrm{~m}$ ). It can potentially solve the problem of broadband and low-latency submarine WCNs [2, 50, 51]. The optical-based underwater WCNs can provide a high-speed data transmission rate (in Gbps) for a moderate transmission range [1, 52, 53]. Moreover, their high-speed transmission also guarantees that optical-based underwater WCNs can be used for many real-time underwater applications, e.g., underwater video transmissions [54-56]. Even though optical-based underwater WCNs offer the great advantage of high data rates for short and medium ranges, its line-of-sight (LoS) requirement might sometimes be difficult to satisfy. In other words, misalignment between the orientation of the optical transmitter and optical receiver might deteriorate the performance of an optical underwater WCN.

\section{Optical Channel Modelling}

Pathloss: The pathloss of optical channel as a function of wavelength $\lambda$ and distance $d$ can be expressed as

$$
P L_{O}(\lambda, d)=\exp (-e(\lambda) d),
$$

where $e(\lambda)$ is known as optical beam extinction coefficient which comprises of scattering coefficient $c(\lambda)$ and absorption coefficient $a(\lambda)$ expressed below;

$$
c(\lambda)=a(\lambda)+s(\lambda) .
$$

The absorption coefficient can be computed as:

$$
a(\lambda)=\frac{d(A(\lambda))}{d r}
$$

where $A(\lambda)=\frac{P_{a}(\lambda)}{P_{i}(\lambda)}$ (known as absorbance), $P_{a}(\lambda)$ is the absorbed power while $P_{i}(\lambda)$ is the incident power. Similarly, the scattering coefficient can computed as:

$$
s(\lambda)=\frac{d(S(\lambda))}{d r},
$$


where $S(\lambda)=\frac{P_{s}(\lambda)}{P_{i}(\lambda)}$ (known as scatterance), and $P_{s}(\lambda)$ is the scattered power.

Noise: The total noise can be expressed as:

$$
P_{\text {noise }}=P_{\text {solar }}+P_{\text {blackbody }},
$$

where $P_{\text {solar }}$ is noise power due to solar while $P_{\text {blackbody }}$ is noise power due to blackbody radiation.

$$
P_{\text {solar }}=A_{R} \Delta \lambda T_{F} R_{\text {solar }}(\pi F O V)^{2}
$$

where $F O V$ is field of view, $A_{R}$ is the receiver area, $\Delta \lambda$ is the optical filter width, $T_{F}$ is the transmissivity of optical filter and solar radiance $R_{\text {solar }}$ is given as:

$$
R_{\text {solar }}=\frac{i_{r} R L_{f} \exp (-K \delta)}{\pi},
$$

where $i_{r}$ is the downwelling irradiance, $R$ is the reflectance of downwelling irradiance, $\delta$ is the underwater depth, $K$ is the coefficient of attenuation and $L_{f}$ is the factor describing the directional dependence of underwater radiance. The noise form blackbody radiation can be expressed as:

$$
P_{\text {blackbody }}=\frac{2 h c^{2} \gamma A_{R} \Delta \lambda T_{F} T_{A}(\pi F O V)^{2}}{\lambda^{5}\left(\exp \left(\frac{h c}{\lambda k T}\right)-1\right)},
$$

where $h$ is a Planck constant, $c$ is the speed of light in water, $\gamma$ is the absorption factor, $T_{A}$ is the transmission and $k$ is the Boltzmann constant.

Channel Impulse Response: According to [57], the CIR of underwater optical communication can be expressed in closed form as

$$
h(t)=C_{1} \Delta t \exp \left(-C_{2} \Delta t\right)+C_{3} \Delta t \exp \left(-C_{4} \Delta t\right), \quad\left(t>t_{0}\right)
$$

where $\Delta t=t-t_{0}, t$ is time scale while $t_{0}=\frac{d}{v}$ is the ratio of distance and velocity of light in water (also known as propagation time), $C_{1}, C_{2}, C_{3}, C_{4}$ are the four variables/parameters which can be found through least square approach.

\section{AIR-WATER WIRELESS COMMUNICATION}

In this section, we provide a brief overview of A-W WCNs. A total of four configurations are considered, namely: 1) acoustic (in water) to RF (in air) [7], 2) acoustic (in water) to optical (in air) [58], 3) light (in water) to RF (in air) [59], and 4) direct light in both mediums (water and air) [60]. Typically, air-water wireless communication can either be indirect or direct depending on the existence of a relay/surface node.

\section{A. Indirect Air-Water Wireless Communication}

In indirect air-water wireless communication systems (also called surface node assisted communication), underwater nodes communicate with surface nodes (also called sink nodes), that are further connected to ground stations via satellite, microwave, or Free-Space Optical (FSO) links. The sink node is equipped with two transceiver interfaces: one for submerged nodes and the other for the water surface nodes. An illustration of such systems is provided in Fig. 2 (a), where different underwater systems are communicating with the surface node via a sink node attached to a ship. Consequently, the addition of an extra hop always leads to increased end-to-end delay, which, in turn, exposes the system to more attacks. Typically, optics in water, and RF in air is considered for end-to-end analysis in indirect A-W communication [59]. However, recently, an experimental study on photoacoustic communication has been conducted in [58], where acoustic is used for underwater and optical communication is used for over water.

\section{Indirect A-W Channel Modelling}

The channel modelling for underwater part (i.e., RF, acoustic and optical) are discussed in Section II while for the aerial part (i.e., RF and Optical) terrestrial model for pathloss, noise and fading are applicable. The main aim of such systems is to analyze the end to end communication.

\section{B. Direct Air-Water Wireless Communication}

Surface nodes are usually attached to large buoys or ships, which can be easily spotted by the enemies/adversaries through the use of Radars or telescopes. Therefore, in order to avoid the need of a surface floating node, a new (potentially revolutionary) paradigm has recently been proposed in [7]. In this first work, direct communication between underwater and over water nodes takes place, where acoustic communication was used for underwater part and RF communication for over water part. The basic idea of direct air-water communication is illustrated in Fig. 2 (b), where the airborne node receives and decodes information from the backscattered RF waves after striking the ripples produced by the acoustic waves on the surface. Similarly, the use of direct light from air to water has been explored in [8] and [61], followed by various works attempting to enhance the performance [60, 62-72].

\section{Direct A-W Channel Modelling}

As per the above discussion (Section III-B) two different systems are considered for direct $\mathrm{A}-\mathrm{W}$ communication. We discuss the channel characteristics of both systems below. System 1: Translation Acoustic-RF (TARF) Communication The end to end channel of TARF comprises of below three parts.

Underwater Part: The pathloss/attenuation in water medium for TARF is given as

$$
P L_{U W}^{T A R F}=\frac{\exp (\gamma r)}{r},
$$

where $r$ is depth and $\gamma$ is the absorption.

Air-Water interface: The attenuation at the A-W interface is given as:

$$
P L_{A-W}^{T A R F}(\omega, t)=\frac{P(\omega, t)}{\rho \omega v},
$$

where $\omega$ is the angular frequency, $t$ is time, $\rho$ is the water density and $P$ is the overall acoustic pressure. Besides, the 


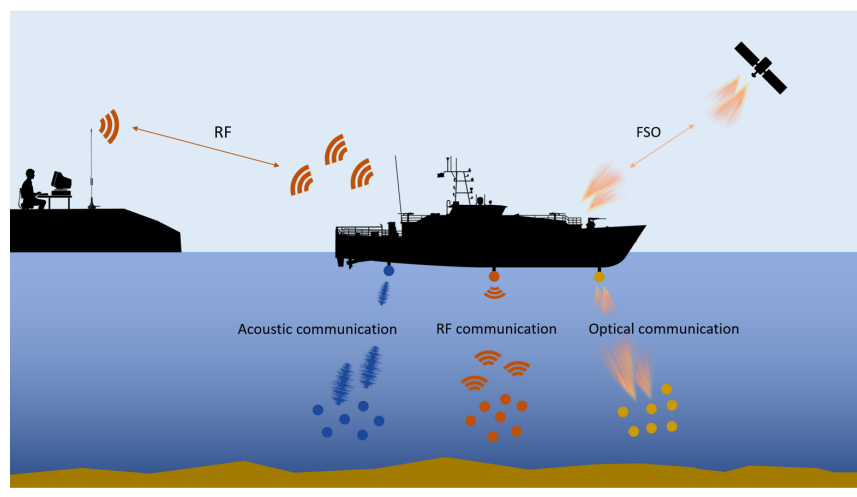

(a)

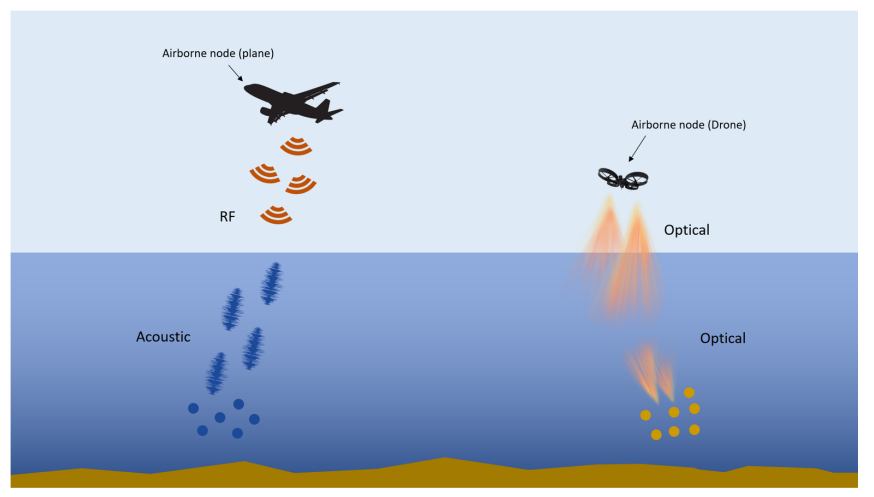

(b)

Fig. 2: Illustration of A-W WCNs. (a) Indirect (Surface node-assisted) communication, (b) Direct communication.

sensed power at this interface for a given incident power $P_{i}$ is given as

$$
P_{(A-W) \underset{\text { sensed }}{\text { TARF }}}=\frac{P_{i}}{\rho v \omega^{2}}
$$

Air Part: The attenuation in this part is given as:

$$
P L_{A}^{T A R F}=\frac{1}{2 d_{0}}
$$

where $d_{0}$ is the distance from airborne node to water surface. System 2: Optical based A-W: The channel of such systems can be characterized through transmittance (can be thought as inverse of pathloss). Following [73], we discuss the transmittance through three regions below.

Air Part: The transmittance in air medium can be expressed as:

$$
T_{\text {Air }}=C_{0}^{T} \exp \left(-\left[\frac{r / r_{0}}{S\left(\frac{2}{R_{e f f}\left(\phi-\psi_{0}\right)}\right)}\right]^{S^{\iota}\left(\frac{2}{R_{e f f}\left(\phi-\psi_{0}\right)}\right)}\right),
$$

where $r$ is the beam deflection distance, $r_{0}$ is the aperture radius $C_{0}^{T}$ is the maximum transmission coefficient at $r=0$, $R_{\text {eff }}($.$) is the effective spot radius, S($.$) is scale function and$ $S^{\circ}($.$) is shape function.$

Surface Part: The transmittance of the surface is given as:

$$
T_{\text {surface }}=\eta_{\text {deflection }} T_{\text {refraction }}
$$

where $\eta_{\text {deflection }}$ denotes efficiency due to deflection of light because of surface roughness, its computation is given in [73], and $T_{\text {refraction }}$ is the total Fresnel transmittance given below.

$$
T_{\text {refraction }}=\frac{T_{\perp}+T_{\|}}{2}
$$

where $T_{\perp}$ is the perpendicular and $T_{\|}$is the parallel components of Fresnel transmittance, which can be computed as

$$
\begin{gathered}
T_{\perp}=1-\frac{\sin ^{2}\left({ }_{i}-_{r}\right)}{\sin ^{2}\left({ }_{i}{ }_{r}\right)} \\
T_{\|}=1-\frac{\tan ^{2}\left({ }_{i}{ }_{r}\right)}{\tan ^{2}\left({ }_{i}{ }_{r}\right)},
\end{gathered}
$$

where ${ }_{i}$ and ${ }_{r}$ are incident and refraction angles respectively. Water Part: The transmittance in water $T_{W}$ can be expressed as

$$
T_{W}=10^{-K / 10}
$$

where $\mathrm{K}$ is the total attenuation coefficient.

Noise: The total noise power is given as

$$
P_{T N}^{O A-W}=E N L+T \frac{P_{N}^{A} \exp (-K \delta)+P_{N}^{W}}{h f_{n}}
$$

Where $E N L$ is excess noise limit, $T$ is the sampling period of detector, $K$ is the attenuation coefficient, $\delta$ is the depth, $h$ is Plank constant, $f_{n}$ is the noise frequency $P_{N}^{A}$ is nose power in air and $P_{N}^{W}$ is noise power in water/sea. The can be expressed as

$$
P_{N}^{A}=B_{s} F O V \pi r^{2} \Delta f
$$

where $B_{s}$ is the sky brightness, $r$ is the radius of virtual telescope and $\Delta f$ is the filter bandwidth and $P_{N}^{W}$ can be expressed as

$$
P_{N}^{W}=R_{\text {solar }} F O V \pi r^{2} \Delta f
$$

where $R_{\text {solar }}$ is the solar radiance given in Eq. 20 .

\section{SECURITY OF UNDERWATER AND A-W WCNS}

In sections II and III, We described the system models, technological enablers, unique challenges of underwater medium, and various system configurations. This should have intrigued the curious reader to anticipate and imagine the various different kinds of attacks that could be launched on underwater and A-W WCNs. For example, one could see that the opticalbased underwater and A-W channels (being highly directive in nature) are least prone to passive eavesdropping attacks by the malicious nodes nearby, especially when compared to their RF and acoustic counterparts. One could also verify that launching a jamming attack on an optical-based system is difficult because the jammer will need to move to the field-of-view of the optical receiver, where the optical jammer will very likely be detected. Similarly, it is not difficult to see that launching 


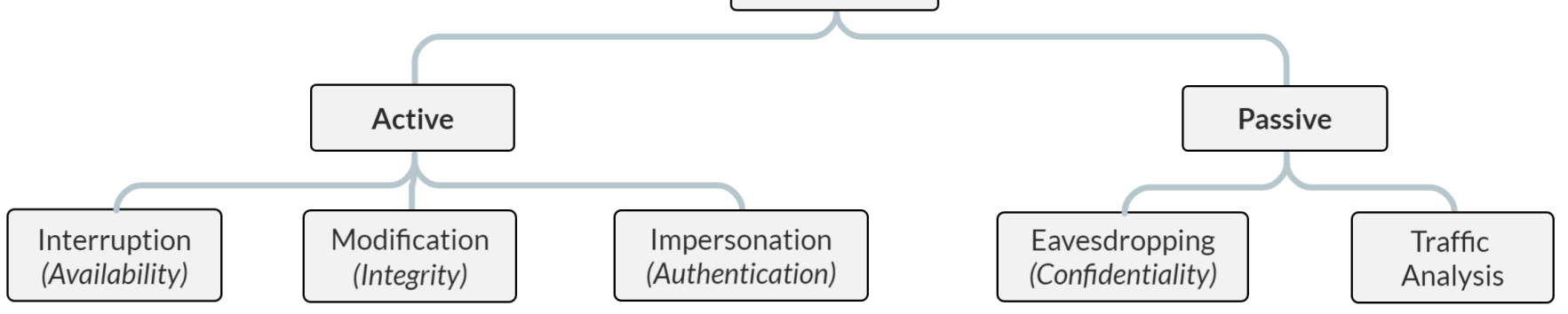

Fig. 3: Broad classification of malicious attacks

\begin{tabular}{|c|c|c|c|c|c|}
\hline \multirow[t]{2}{*}{ Security Property } & \multicolumn{3}{|c|}{ Underwater Wireless Communication } & \multicolumn{2}{|c|}{ Air-Water Wireless Communication } \\
\hline & $\mathrm{RF}$ & Acoustic & Optical & Indirect & Direct \\
\hline Confidentiality & - & 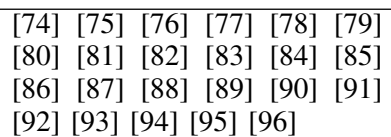 & $\begin{array}{l}{[9][97][98][99][100]} \\
{[101][102][103][104]} \\
{[105][106]}\end{array}$ & [107] [108] [109] [110] [111] & [112] [73] [113] [114] \\
\hline Integrity & - & {$[115][76][116][117][93]$} & - & - & - \\
\hline Authentication & - & $\begin{array}{lllll}{[74]} & {[118]} & {[75]} & {[115]} & {[119]} \\
{[120]} & {[121]} & {[76]} & {[79]} & {[122]} \\
{[123]} & {[124]} & {[80]} & {[125]} & {[126]} \\
{[127]} & {[116]} & {[81]} & {[82]} & {[86]} \\
{[128]} & {[129]} & {[130]} & {[131]} & {[88]} \\
{[132]} & {[93]} & {[133]} & {[94]} & {[134]} \\
{[95]} & {[96]} & {[135]} & {[136]} & {[137]}\end{array}$ & $\begin{array}{l}{[100][101][102][103]} \\
{[104][105][106]}\end{array}$ & - & [112] [73] [113] [114] \\
\hline Availability & - & $\begin{array}{l}{[118][138][115][139][140]} \\
{[141][123][124][125][142]} \\
{[83][84][128][128][91][92]} \\
{[133][137]}\end{array}$ & - & - & - \\
\hline
\end{tabular}

TABLE III: Current state-of-the-art in underwater and air-water wireless communication: each work has been mapped to one of the four main properties of information security.

an impersonation (stealth) attack on RF-based underwater and A-W WCNs is relatively straightforward; mainly because typically it is much easier to localize a malicious acoustic or optical source (being audible or visible, intermittently).

This brings us to the main contribution of this paper where we provide a thorough and up-to-date survey of the relevant papers in the field addressing the essential properties of information security as shown in Table III. However, before we delve into the security of underwater and A-W communications, it is imperative to provide a quick background on the domain of information security.

\section{A. A primer on information security}

Information security aims to protect information exchanged between legitimate parties. Any communication system with the provisioning of information security should ensure that the following properties (also known as four fundamental properties of information security) are preserved:

- Confidentiality: ensures secrecy of transmitted information.

- Authenticity: ensures legitimacy of exchanging parties.

- Integrity: ensures that information exchanged between legitimate nodes have not been modified.
- Availability: ensures the availability of the system for legitimate nodes.

To preserve these properties, two broad approaches are adopted: traditional cryptography and Physical Layer Security (PLS), which we briefly discuss next.

1) Traditional cryptography: Typically, to establish secure communication, some cryptographic measures need to be achieved, where legitimate nodes jointly solve a complex mathematical problem (e.g., elliptic curves) to establish shared secrets, that can then be used to preserve confidentiality, authentication and integrity. Crypto-based systems can be divided into two main classes, symmetric and asymmetric key cryptography.

- Symmetric key: also known as private-key cryptography is an approach for encrypting and decrypting information with a pre-shared secret key. The most popular symmetric key algorithm is Advanced Encryption Standard (AES).

- Asymmetric key: also known as public-key cryptography, is an approach for encrypting and decrypting information using two keys, a public key (known to everyone) and private (known to owner only). Messages encrypted by one key can only be decrypted by the other. The most popular asymmetric key algorithms are RSA [143] and DH [144]. 
However, recent advances in computational resources and quantum computing, may potentially jeopardize these cyrptobased solutions [145-148]. This has motivated the community to explore alternative approaches, a popular one is: Physical Layer Security.

2) Physical Layer Security (PLS): PLS is increasingly becoming an attractive area of research due to its promising performances in many wireless communication systems [149158]. PIS exploits the random nature of the physical layer for security purposes, which not only enhances security, but also greatly improve efficiency. In wireless communication, randomness is introduced due to two main sources: wireless channel and hardware. The randomness in the wireless channel is introduced due to random noise and random multi-paths arrival, which forcefully randomizes some processes such as Channel Impulse Response (CIR), Channel Frequency Response (CFR), and Received Signal Strength (RSS). On the other hand, randomness in hardware is introduced due to uncontrollable impurities added during the manufacturing process.

Like traditional cryptography, PLS attempts to preserve a number of important security properties as illustrated in fig. 7.

Physical layer confidentiality provides security to information in-transit. in other words, this mechanism tackles the eavesdropping attacks where the eavesdropper is in listening mode. The basis of this mechanism was first introduced by Wyner in his seminal work [159]. The secrecy rate and the probability of leakage or secrecy outage (which depends on information-theoretic bounds) are the two main performance metrics for physical layer confidentiality. The secrecy rate can be enhanced through optimal resource allocation [152] (such as depicted in Fig. 4, taken from one of our previous works [87]) and/or artificial noise generation [160], where noisy signals are generated in the null space of the legitimate users. Through similar procedures, secrecy outages can be minimized. Resources such as carrier power, carrier selection, relay selection, and user selection are exploited for physical layer confidentiality [161], [151].

Physical layer authentication is a systematic procedure that verifies the legitimacy of the transmitter node based on the characteristics of the physical layer, such as device fingerprints or features. Physical layer authentication generally involves two steps: feature estimation and testing [162]. This mechanism requires a feature that is random in nature and independent for distinct transmitters. The reported features can be classified into medium/channel-based features and hardware-based features (or RF-fingerprints). CIR [153], CFR [163], RSS [164] are examples of channel-based features. In-phase/Quadrature (I/Q) imbalance [165] and carrier offsets (CO) [166], [167] are examples of hardware features. Error probabilities (false alarm and miss detection which also produce Receiver Operating Characteristics (ROC) curves (depicted in Fig. 5, taken from one of our previous works [167] )), Kullback-Leibler Divergence (KLD) [167] [168] and Jensen-Shannon Divergence (JSD) [169] are key performance

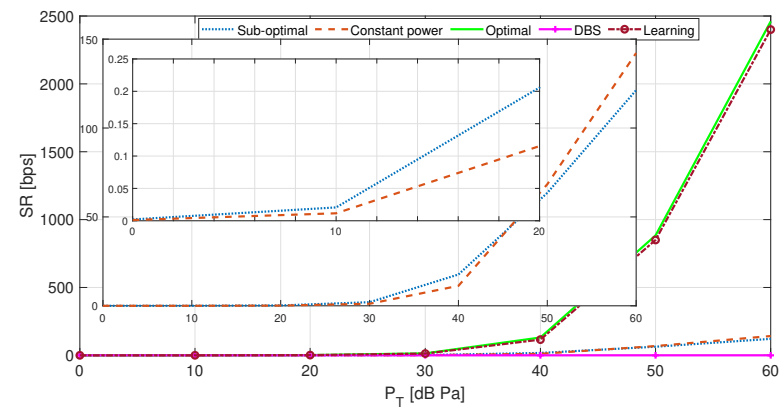

Fig. 4: Secrecy rate (SR) vs total power budget $P_{t}$ of underwater acoustic communication system considered in [87]. The performance of proposed optimal scheme is compared against other proposed schemes (i.e., learning and sub-optimal) and baseline schemes (i.e., Depth Based Selection (DBS) and constant power).

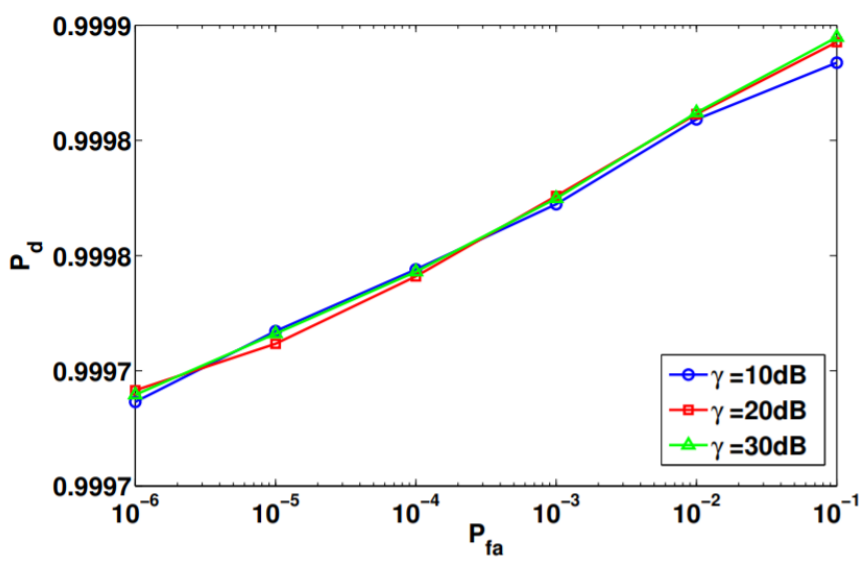

Fig. 5: ROC: probability of detection $P_{d}$ against probablity of false alarm $P_{f a}$ for various values of SNR $\gamma$ [167].

metrics to evaluate physical layer authentication mechanisms. Shared secret key generation is a process where a pair of legitimate users extract a secret key from commonly shared time-varying sources (features/attributes of the physical layer). This mechanism aims to avoid the dependency on preshared secret keys. This mechanism requires a reciprocal time-varying sources or features among the legitimate users to extract secret keys. Feature estimation, quantization, key reconciliation and privacy amplification are standard steps used to extract the keys. Mutual information, key generation rate, key disagreement rate (illustrated in Fig. 6, taken from one of our previous works [155]) bit-match rate, burst-match rate, and decipher probability are key performance metrics used to evaluate this mechanism's performance. RSS, CIR [170], CFR [171], CO [155] are examples of features that can be used to generate secret key. The generated secret keys can then be used to provide both confidentiality (by using any symmetric cryptography approaches) and authentication (by using generated keys as transmit device fingerprint [172]) in communication systems. 


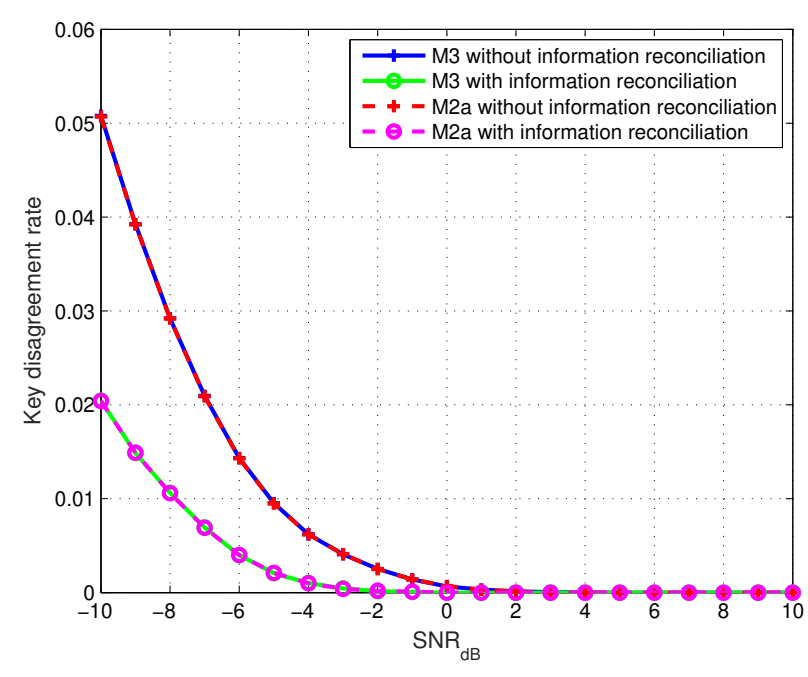

Fig. 6: Key disagreement rate against SNR (in dB) for two proposed $\mathrm{CFO}$ models (i.e., $\mathrm{M} 2 \mathrm{a}$, where $\mathrm{CFO}$ is time-varying and memory-full and M3, where CFO is time varying and memoryless) [155].

3) Attack Types: Common malicious attacks jeopardizing the above security properties (i.e., confidentiality, authentication, integrity, availability) can be broadly classified as active and passive attacks. In active attacks, a malicious node transmits malicious signals to obtain illegal access or disrupt ongoing communication. In passive attacks, a malicious node does not transmit any signals but listens passively to the legitimate nodes' ongoing communication. A broad classification of attacks, and what security properties they compromise, is illustrated in Fig. 3. Examples of these attacks, include:

- Spoofing: also known as impersonation, where a malicious node claims identity of a legitimate node.

- Replay: in this attack, a malicious node re-uses intercepted legitimate messages.

- Sybil: in this attack, a malicious node claims identities of many legitimate nodes.

- TSA: in this attack, a malicious node tampers with legitmate nodes' time synchronization, which is a fundamental requirement for localization purpose. The goal of the malicious node is to convince some nodes that their neighborsâ clocks are at a different time than they actually are.

- Wormhole: two or more malicious nodes jointly launch this attack. At one point of the network, attacker receives packets and tunnel them to another point in the network and then replay them from the that point. This attack typically disrupts the routing procedure of the network.

- Blackhole: in this attack, a malicious node advertises convincing parameters to the legitimate nodes in order to become a forwarding node in their routing path. The goal of the attacker is to create a hole in the network when incoming packets can be dropped.

- Jamming: in this attack, a malicious node bombard legit- imate nodes with excessive amount of signals potentially making it unavailable to process anything else. Jamming is typically either active jamming and reactive jamming. In former, a jammer node produces jamming signals continuously while in later, a jammer transmits whenever communication between legitimates parties is detected.

- Eavesdropping: in this attack, a malicious node is interested in the communication between legitimate parties and tries to listen and decode intercepted traffic.

- DoS: in this attack. a malicious node attempts to prevent the legitimate nodes from accessing network resources. Typically, DoS is achieved through jamming and flooding. Therefore, Jamming, Wormhole, blackhole are considered as DoS attacks.

\section{B. Security of Underwater Wireless Communication}

Fig. 8 illustrates a scenario where adversaries present in the close vicinity of legitimate nodes perform underwater malicious attacks. The attacks can be active or passive.

Recall from Section-II that underwater wireless communication can be accomplished using RF, acoustic and optical communication technologies. Hence, we now discuss the most popular attacks on each underwater communication technology.

1) Security of RF-based underwater WCN: To the best of our knowledge, there are no recent advances on RF-based underwater $\mathrm{WCN}$ due to their shortcomings discussed in section II-A. Hence, we found no work studying attacks on such systems.

2) Security of acoustic-based underwater WCN: Underwater acoustic communication became an active research area in the last few decades, which resulted in quantitative and qualitative research on the security of underwater acoustic communication. We summarize the state-of-the-art security in underwater acoustic communication in terms of confidentiality, authentication, and integrity, in Tables IV, V \& VI. Table IV summarizes the relevant works published before 2019; Table V summarizes relevant works published during the period 2019-20; while Table VI summarizes relevant works published from 2021 to date. The most common scenarios assumed for underwater communications are multi underwater nodes (more than two legitimate nodes and one or more malicious node) and three underwater nodes (i.e., two legitimate nodes and a malicious node). Furthermore, single carrier, multi-carrier (i.e., Orthogonal Frequency Division Multiplexing (OFDM)), Line-of-Sight (LoS), and non-LoS underwater acoustic communications are exploited for security purposes. In short, various system configurations are judged for their strength and weaknesses against attacks. Some configurations will do better than others, for each kind of attack. The counter-mechanism strategies borrow tools from cryptography, optimization theory, game theory, control theory, probability theory and machine learning to equivocate/confuse a passive malicious node, or to partially recover data amid an active jamming or spoofing attack by a malicious node. 


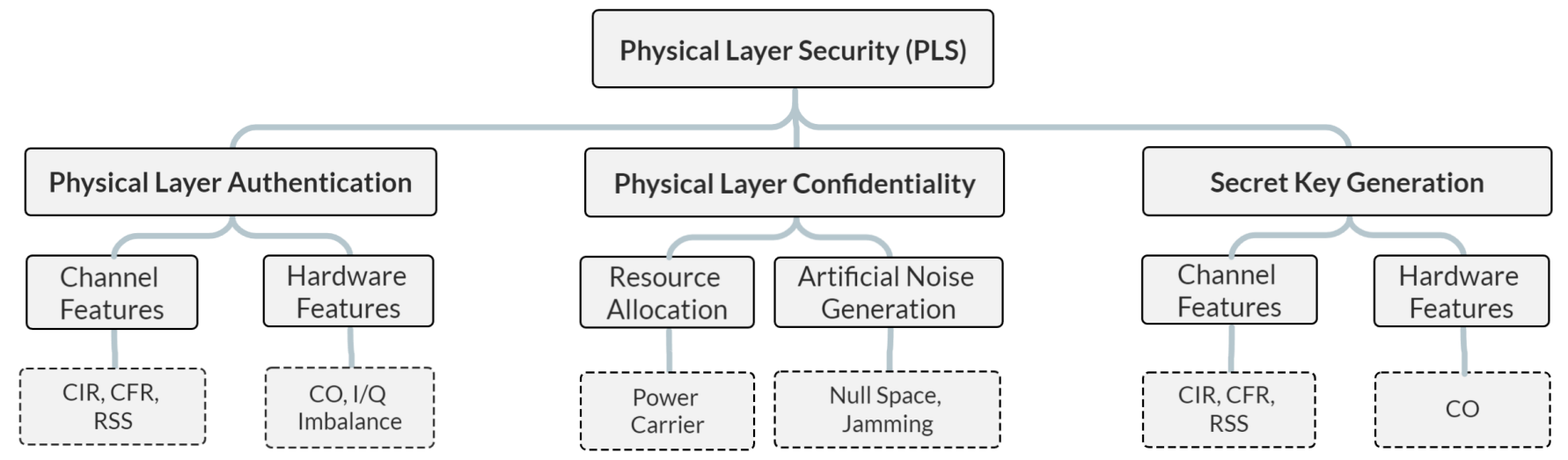

Fig. 7: Security properties preserved by PLS. The Figure also lists examples of features that can be used provide these properties.

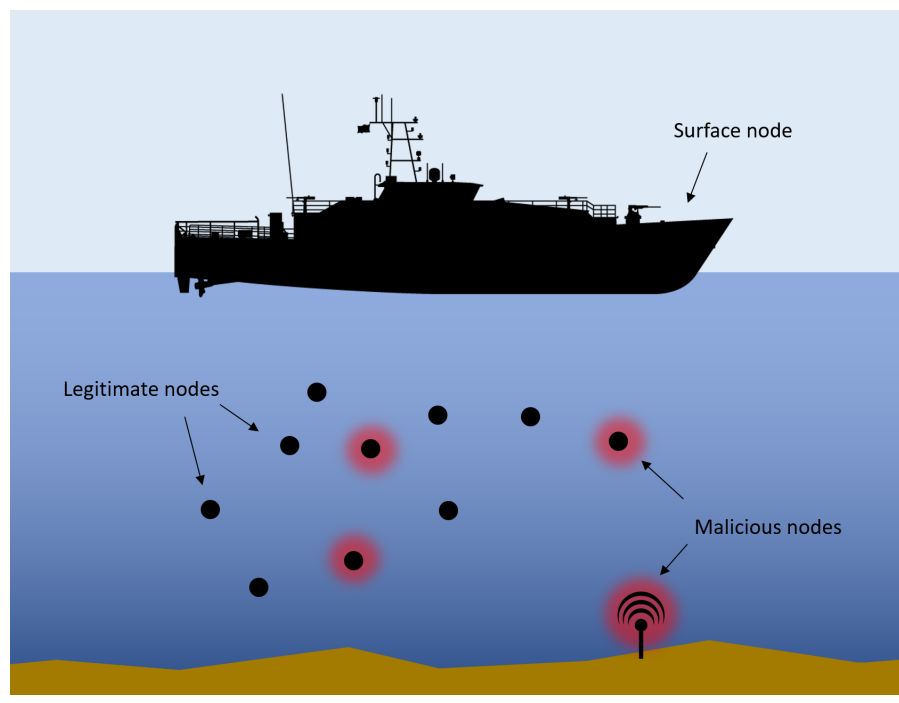

Fig. 8: Illustration of a scenario where malicious nodes present in underwater launch active and passive attacks. The malicious seabed node is depicted as an active malicious node, while the other is a passive malicious node.

As shown in Tables IV, V \& VI, the most common attacks considered in underwater acoustic communication are spoofing (also known as impersonation), eavesdropping, and Denial of Service (DoS). Variants of these attacks are also sometimes considered, such as the replay, sybil, masquerade, wormhole, Time Synchronization Attack (TSA), Blackhole and time delay attacks, which are variants of the spoofing attacks. Jamming, Wormhole and Blackhole are also considered DoS attacks [173]. Tables IV, V \& VI also provide details about some countermeasures that have been shown to resist these attacks.

3) Security of optical-based underwater WCN: Opticalbased underwater WCN is generally considered a relatively more secure communication approach (compared to its acoustic and RF-based counterparts) due to the high directivity of the light beam [174]. However, an eavesdropper located in a close vicinity to the transmitter and receiver might disturb the information transmission by partially blocking the transmitting light-beam [57]. Indeed, the eavesdropper might require a sophisticated device such as a beam splitter as the laser beam near the transmitter node becomes narrow. Therefore, the authors in [9] experimentally illustrated some vulnerabilities on underwater wireless optical communication. They use reflecting mirrors between communication paths to measure any leakage towards the eavesdropper [97]. Similarly, authors in [98] used diffraction grating to tap the optical channel. Recently, the experimental work in [99] studied the chaotic encryption in $50 \mathrm{~m} / 5 \mathrm{Gbps}$ using $450 \mathrm{~nm}$ laser to provide confidentiality. Furthermore, the laser beam experiences divergence due to the scattering phenomenon [175]. In principle, the light wave strikes an aerosol (i.e. dust particles or gas molecules) and scatters into several light beams in different directions [176]. Therefore, it is possible that an eavesdropper located near the receiver can capture the scattered photons and hence intercepts the legitimate information transmission.

On the other hand, recently, Quantum Key Distribution (QKD) has been explored theoretically based on opticalbased underwater WCN [101, 103-106]. QKD is a promising mechanism where secret keys are generated based quantum mechanical properties, such as entanglement [100-106]; the no-cloning theorem ${ }^{1}$ [177] then guarantees that these keys cannot be intercepted. QKD is divided into Discrete Variable QKD (DV-QKD) [178-180] and Continuous Variable QKD (CV-QKD) [181-184], where the latter has recently been preferred due to easy implementation and higher channel capacity [101, 103-106].

\section{Security of Air-Water Wireless Communication}

The security needs of the A-W WCNs has received very little attention by the research community so far. A typical scenario of malicious attacks in A-W WCNs is illustrated in Fig. 9. There are only a handful of papers that discuss

\footnotetext{
${ }^{1}$ In Quantum mechanics, the no-cloning theorem states that it is not possible to make a copy of a Quantum state as copying it will entail observing it, and observing it will inevitably destroy it.
} 


\begin{tabular}{|c|c|c|c|c|}
\hline Ref. & $\begin{array}{l}\text { System } \\
\text { Architecture }\end{array}$ & Attacks considered & Countermeasures proposed & $\begin{array}{l}\text { Security Properties ad- } \\
\text { dressed }\end{array}$ \\
\hline [74] (2008) & $\begin{array}{lr}\text { Three nodes } & \text { (two } \\
\text { legitimate } & \text { and } \\
\text { one } & \text { malicious } \\
\text { nodes) Underwater } & \text { Undic } \\
\text { Acoustic } & \text { Sensor } \\
\text { Network (UWASN) }\end{array}$ & $\begin{array}{l}\text { Spoofing and Eaves- } \\
\text { dropping }\end{array}$ & $\begin{array}{l}\text { Shared secret key generation. } \\
\text { Feature:Envelope of tone signal. Robust } \\
\text { Secure Fuzzy Information Reconciliation } \\
\text { (RSFIR) }\end{array}$ & $\begin{array}{l}\text { Confidentiality and Authen- } \\
\text { tication }\end{array}$ \\
\hline [118] (2010) & $\begin{array}{l}\text { Multi-nodes single- } \\
\text { hop UWASN }\end{array}$ & Wormhole & $\begin{array}{l}\text { Direction of arrival (DoA) for each node } \\
\text { is estimated to make the network resilient } \\
\text { to wormhole attack during neighbour dis- } \\
\text { covery for suit of protocols }\end{array}$ & $\begin{array}{l}\text { Authentication and Avail- } \\
\text { ability }\end{array}$ \\
\hline [138] (2011) & $\begin{array}{l}\text { Multi-nodes multi- } \\
\text { hop UWASN }\end{array}$ & DoS (Jamming) & Multi-path routing & Availability \\
\hline [75] (2011) & $\begin{array}{l}\text { Underwater acoustic } \\
\text { network of AUVs }\end{array}$ & $\begin{array}{l}\text { Spoofing and Eaves- } \\
\text { dropping }\end{array}$ & Crypto-based suit & $\begin{array}{l}\text { Confidentiality, Authentica- } \\
\text { tion and Integrity }\end{array}$ \\
\hline [115] (2011) & $\begin{array}{l}\text { Underwater acoustic } \\
\text { network of AUVs }\end{array}$ & Spoofing and DoS & $\begin{array}{l}\text { Crypto-based scheme: Secure FlOOD (Se- } \\
\text { FLOOD), where FLOOD is a network } \\
\text { discovery protocol }\end{array}$ & $\begin{array}{l}\text { Authentication, Integrity } \\
\text { and Availability }\end{array}$ \\
\hline [139] (2012) & $\begin{array}{l}\text { Multi-nodes multi- } \\
\text { hop UWASN }\end{array}$ & Jamming & $\begin{array}{l}\text { Underwater Jamming Detection Protocol } \\
\text { (UWJDP): packet send ratio, packet deliv- } \\
\text { ery ratio and energy consumption amount } \\
\text { are used. }\end{array}$ & Availability \\
\hline [119] (2013) & $\begin{array}{l}\text { Multi-nodes multi- } \\
\text { hop UWASN }\end{array}$ & Spoofing & $\begin{array}{l}\text { Crypto-based scheme: Digital signatures } \\
\text { generated using three schemes-ECDSA, } \\
\text { BLS and ZSS. }\end{array}$ & Authentication \\
\hline$[120](2013)$ & $\begin{array}{l}\text { Multi-nodes multi- } \\
\text { hop UWASN }\end{array}$ & Sybil & $\begin{array}{l}\text { Received packet and time rates, disposed } \\
\text { and identification packet rates are used. }\end{array}$ & Authentication \\
\hline$[140](2015)$ & $\begin{array}{l}\text { Two legitimate nodes } \\
\text { UWASN with multi- } \\
\text { ple jammers }\end{array}$ & Jamming & $\begin{array}{l}\text { No countermeasures are provided. De- } \\
\text { tailed experimental results are demon- } \\
\text { strated. The experiments are conducted in } \\
\text { Mansfield Hollow Lake, CT, USA }\end{array}$ & Availability \\
\hline [141] (2015) & $\begin{array}{l}\text { CSMA-based } \\
\text { multiple-nodes } \\
\text { single-hop UWASN }\end{array}$ & Jamming & $\begin{array}{l}\text { Static and dynamic jamming games. Nash } \\
\text { equilibrium for static game and re- } \\
\text { enforcement learning based solution for } \\
\text { dynamic game are proposed }\end{array}$ & Availability \\
\hline [121] (2015) & $\begin{array}{l}\text { Multi-nodes single- } \\
\text { hop UWASN }\end{array}$ & Spoofing & $\begin{array}{l}\text { Game theoretic approach: Nash Equilib- } \\
\text { rium is derived for zero-sum game be- } \\
\text { tween a spoofer and sensors }\end{array}$ & Authentication \\
\hline [76] (2015) & $\begin{array}{l}\text { Multi-nodes single- } \\
\text { hop UWASN }\end{array}$ & $\begin{array}{l}\text { Spoofing and Eaves- } \\
\text { dropping }\end{array}$ & $\begin{array}{l}\text { Crypto-based schemes: symmetric and } \\
\text { asymmetric keys based encryption and au- } \\
\text { thentication }\end{array}$ & $\begin{array}{l}\text { Confidentiality, Authentica- } \\
\text { tion and Integrity }\end{array}$ \\
\hline [77] (2016) & $\begin{array}{l}\text { Multi-nodes } \\
\text { UWASN }\end{array}$ & Eavesdropping & $\begin{array}{l}\text { No countermeasures: probability of eaves- } \\
\text { dropping is calculated using stochastic ge- } \\
\text { ometry }\end{array}$ & Confidentiality \\
\hline [78] (2016) & $\begin{array}{l}\text { OFDM-based three- } \\
\text { nodes UWASN }\end{array}$ & Eavesdropping & $\begin{array}{l}\text { Jamming is used to enhance the secrecy } \\
\text { rate }\end{array}$ & Confidentiality \\
\hline [79] (2016) & $\begin{array}{l}\text { OFDM based three } \\
\text { nodes UWASN }\end{array}$ & $\begin{array}{l}\text { Spoofing and Eaves- } \\
\text { dropping }\end{array}$ & $\begin{array}{l}\text { Feature: Amplitudes of Channel Fre- } \\
\text { quency Response (CFR). Experimentation } \\
\text { in Mansfield Hollow Lake, Connecticut, } \\
\text { USA. Amplitudes of CFR is estimated and } \\
\text { then quantized using CDF based quantiza- } \\
\text { tion. BCH codes are used for key recon- } \\
\text { ciliation }\end{array}$ & $\begin{array}{l}\text { Confidentiality and Authen- } \\
\text { tication }\end{array}$ \\
\hline [122] (2017) & $\begin{array}{l}\text { Multi-nodes multi- } \\
\text { hop UWASN }\end{array}$ & Spoofing (Forgery) & $\begin{array}{l}\text { Secure routing: two-way authentication } \\
\text { through use of digital signatures }\end{array}$ & Authentication \\
\hline [123] (2017) & $\begin{array}{l}\text { Multi-nodes multi- } \\
\text { hop UWASN }\end{array}$ & Wormhole & Secure localization scheme & $\begin{array}{l}\text { Authentication and Avail- } \\
\text { ability }\end{array}$ \\
\hline [124] (2017) & $\begin{array}{l}\text { Multi-nodes multi- } \\
\text { hop UWASN }\end{array}$ & Wormhole & Agent-based Secure routing scheme & $\begin{array}{l}\text { Authentication and Avail- } \\
\text { ability }\end{array}$ \\
\hline [80] (2018) & $\begin{array}{l}\text { OFDM-based } \\
\text { UWASN }\end{array}$ & $\begin{array}{l}\text { Spoofing and Eaves- } \\
\text { dropping }\end{array}$ & $\begin{array}{l}\text { Shared secret key generation. Feature: } \\
\text { Phases of CFR. Deterministic quantiza- } \\
\text { tion approach, called Virtual Phase Shift } \\
\text { (VPN) is used for key generation }\end{array}$ & $\begin{array}{l}\text { Confidentiality and Authen- } \\
\text { tication }\end{array}$ \\
\hline [126] (2018) & $\begin{array}{l}\text { Multi-nodes single- } \\
\text { hop UWASN }\end{array}$ & Spoofing & $\begin{array}{l}\text { Physical layer security: A novel three fea- } \\
\text { tures based authentication }\end{array}$ & Authentication \\
\hline$[127](2018)$ & $\begin{array}{ll}\text { Three } & \text { nodes } \\
\text { UWASN } & \end{array}$ & Spoofing & $\begin{array}{l}\text { Reinforcement-learning-based and deep- } \\
\text { reinforcement-learning-based authentica- } \\
\text { tion mechanisms: average power and de- } \\
\text { lay in channel power delay profile are used } \\
\text { as features }\end{array}$ & Authentication \\
\hline$[125](2018)$ & $\begin{array}{l}\text { Two legitimate and } \\
\text { a malicious acoustic } \\
\text { nodes }\end{array}$ & Jamming & $\begin{array}{l}\text { Q-learning based scheme: Transmit power } \\
\text { and node mobility is used to mitigate } \\
\text { jamming }\end{array}$ & Availability \\
\hline
\end{tabular}

TABLE IV: Summary of the reported security work in underwater acoustic communication systems (Upto 2019). 


\begin{tabular}{|c|c|c|c|c|}
\hline Ref. & $\begin{array}{l}\text { System } \\
\text { Architecture }\end{array}$ & Attacks considered & Countermeasures proposed & $\begin{array}{l}\text { Security properties ad- } \\
\text { dressed }\end{array}$ \\
\hline [142] (2019) & $\begin{array}{l}\text { Multi-nodes } \\
\text { UWASN with } \\
\text { single jammer }\end{array}$ & Jamming & $\begin{array}{l}\text { Channel access model based on cross- } \\
\text { layer design }\end{array}$ & Availability \\
\hline [116] (2019) & $\begin{array}{l}\text { Multi-nodes single- } \\
\text { hop UWASN }\end{array}$ & Spoofing & $\begin{array}{l}\text { Cooperative authentication based on stat- } \\
\text { ics of estimated channel characteristics }\end{array}$ & Authentication and Integrity \\
\hline [81] (2019) & $\begin{array}{l}\text { Three-nodes } \\
\text { UWASN }\end{array}$ & $\begin{array}{l}\text { Spoofing and Eaves- } \\
\text { dropping }\end{array}$ & $\begin{array}{l}\text { CIR features: } \mathrm{L}_{2} / \mathrm{L}_{0} \text { norm, a smooth } \\
\text { sparseness measure and the root-mean- } \\
\text { square delay spread. Thousands of } \\
\text { channel-probe signals are used to extract } \\
\text { the CIR features }\end{array}$ & $\begin{array}{l}\text { Confidentiality and Authen- } \\
\text { tication }\end{array}$ \\
\hline [82] (2019) & $\begin{array}{l}\text { Three-nodes } \\
\text { UWASN }\end{array}$ & $\begin{array}{l}\text { Spoofing and Eaves- } \\
\text { dropping }\end{array}$ & $\begin{array}{l}\text { CIR features: } \mathrm{L}_{2} / \mathrm{L}_{0} \text { norm, channel } \\
\text { sparseness, channel energy, root-mean- } \\
\text { square delay spread, mamximum delay } \\
\text { spread and sum of successive delays. } \\
\text { Thousands of channel-probe signals are } \\
\text { used to extract the CIR features }\end{array}$ & $\begin{array}{l}\text { Confidentiality and Authen- } \\
\text { tication }\end{array}$ \\
\hline [83] (2020) & $\begin{array}{l}\text { Multi-nodes } \\
\text { UWASN with } \\
\text { single jammer }\end{array}$ & Jamming & Resource allocation scheme & $\begin{array}{l}\text { Confidentiality and Avail- } \\
\text { ability }\end{array}$ \\
\hline [84] (2020) & $\begin{array}{l}\text { Multi-nodes } \\
\text { UWASN with } \\
\text { multiple jammers }\end{array}$ & Jamming & Resource allocation scheme & $\begin{array}{l}\text { Confidentiality and Avail- } \\
\text { ability }\end{array}$ \\
\hline$[85](2020)$ & $\begin{array}{l}\text { Two legitimate nodes } \\
\text { and a jammer }\end{array}$ & Jamming & $\begin{array}{l}\text { Game theory: optimal strategies are de- } \\
\text { rived for both nodes (defender and at- } \\
\text { tacker) to estimate attacker's location. } \\
\text { Bayesian Nash Equilibrium (BNE) is de- } \\
\text { rived for considered Bayesian Jamming } \\
\text { game }\end{array}$ & $\begin{array}{l}\text { Confidentiality and Avail- } \\
\text { ability }\end{array}$ \\
\hline [86] (2020) & $\begin{array}{l}\text { Multi-nodes single- } \\
\text { hop UWASN }\end{array}$ & $\begin{array}{l}\text { Spoofing and Eaves- } \\
\text { dropping }\end{array}$ & $\begin{array}{l}\text { Crypto-based schemes: compressive sens- } \\
\text { ing based homomorphism encryption and } \\
\text { trust scheme }\end{array}$ & $\begin{array}{l}\text { Confidentiality and Authen- } \\
\text { tication }\end{array}$ \\
\hline$[128](2020)$ & $\begin{array}{l}\text { Multi-nodes multi- } \\
\text { hop UWASN }\end{array}$ & Wormhole & $\begin{array}{l}\text { Secure and energy efficient cooperative } \\
\text { routing }\end{array}$ & $\begin{array}{l}\text { Authentication and Avail- } \\
\text { ability }\end{array}$ \\
\hline [129] (2020) & $\begin{array}{l}\text { Three-nodes (two le- } \\
\text { gitimate and a mali- } \\
\text { cious) LoS UWASN }\end{array}$ & Spoofing & $\begin{array}{l}\text { Angles-of-arrival (azimuth and elevation) } \\
\text { is used as features for physical layer au- } \\
\text { thentication }\end{array}$ & Authentication \\
\hline$[130](2020)$ & $\begin{array}{l}\text { Two Legitimate and } \\
\text { a malicious nodes }\end{array}$ & Spoofing & $\begin{array}{l}\text { Maximum time-reversal resonating } \\
\text { strength as a physical layer feature is } \\
\text { used for authentication }\end{array}$ & Authentication \\
\hline [131] (2020) & $\begin{array}{l}\text { Multi-nodes multi- } \\
\text { hop UWASN }\end{array}$ & Spoofing & Time-series data with fuzzy logic & Authentication \\
\hline [117] (2020) & $\begin{array}{l}\text { Multi-nodes multi- } \\
\text { hop UWASN }\end{array}$ & $\begin{array}{l}\text { Spoofing (replay at- } \\
\text { tack) }\end{array}$ & $\begin{array}{l}\text { Countermeasures based on time stamp and } \\
\text { Hash value of a node }\end{array}$ & Integrity \\
\hline [87] (2020) & $\begin{array}{l}\text { Multi-nodes multi- } \\
\text { hops UWASN }\end{array}$ & Eavesdropping & $\begin{array}{l}\text { Physical layer confidentiality: resource } \\
\text { optimization for enhancing secrecy rate of } \\
\text { the system }\end{array}$ & Confidentiality \\
\hline [88] (2020) & $\begin{array}{l}\text { Multi-nodes single- } \\
\text { hop UWASN }\end{array}$ & $\begin{array}{l}\text { Spoofing and Eaves- } \\
\text { dropping }\end{array}$ & $\begin{array}{l}\text { Secret key generation: multi-party secret } \\
\text { key generation scheme using bi-linear } \\
\text { mapping approach }\end{array}$ & $\begin{array}{l}\text { Confidentiality and Authen- } \\
\text { tication }\end{array}$ \\
\hline [89] (2020) & $\begin{array}{l}\text { Two legitimate nodes } \\
\text { with single jammer }\end{array}$ & Jamming & $\begin{array}{l}\text { Game theoretic approach: Jammer and } \\
\text { legitimate transmitter are considered as } \\
\text { rational player of zero-sum game. Legiti- } \\
\text { mate transmitter exploits the packet-level } \\
\text { coding at a cost of its battery energy to } \\
\text { protect its transmission from jamming }\end{array}$ & $\begin{array}{l}\text { Confidentiality and Avail- } \\
\text { ability }\end{array}$ \\
\hline [90] (2020) & $\begin{array}{l}\text { Multi-nodes single- } \\
\text { hop UWASN }\end{array}$ & Eavesdropping & $\begin{array}{l}\text { Resource allocation: two different opti- } \\
\text { mization models (one minimizes eaves- } \\
\text { dropping risks while other maximizes net- } \\
\text { work life time under constraint on eaves- } \\
\text { dropping risks) are proposed to counter } \\
\text { attacks }\end{array}$ & Confidentiality \\
\hline [91] (2020) & $\begin{array}{l}\text { Multi-nodes multi- } \\
\text { hop UWASN }\end{array}$ & $\begin{array}{l}\text { Eavesdropping and } \\
\text { Active jamming }\end{array}$ & $\begin{array}{l}\text { No countermeasures are provided but ma- } \\
\text { chine learning algorithms are used to se- } \\
\text { lect optimal node for attacking }\end{array}$ & $\begin{array}{l}\text { Confidentiality and Avail- } \\
\text { ability }\end{array}$ \\
\hline
\end{tabular}

TABLE V: Summary of the reported security work in underwater acoustic communication systems (2019-2020). 


\begin{tabular}{|c|c|c|c|c|}
\hline Ref. & $\begin{array}{l}\text { System } \\
\text { Architecture }\end{array}$ & Attacks considered & Countermeasures proposed & $\begin{array}{lll}\text { Security } & \text { Properties ad- } \\
\text { dressed } & & \\
\end{array}$ \\
\hline [92] (2021) & $\begin{array}{l}\text { Two legitimate nodes } \\
\text { with a jammer }\end{array}$ & Jamming & $\begin{array}{l}\text { Network geometry based Game theoreti- } \\
\text { cal framework }\end{array}$ & $\begin{array}{l}\text { Confidentiality and Avail- } \\
\text { ability }\end{array}$ \\
\hline [132] (2021) & $\begin{array}{l}\text { Internet of Underwa- } \\
\text { ter Things (IoUT) }\end{array}$ & $\begin{array}{l}\text { Spoofing (Time Syn- } \\
\text { chronization Attack } \\
\text { (TSA)) }\end{array}$ & $\begin{array}{l}\text { Mix-integer programming probelm is for- } \\
\text { mulated and solved via expectation maxi- } \\
\text { mization and constrained variable thresh- } \\
\text { old rounding methods }\end{array}$ & Authentication \\
\hline [93] (2021) & $\begin{array}{l}\text { Two legitimate and a } \\
\text { malicious nodes }\end{array}$ & $\begin{array}{l}\text { Spoofing and Eaves- } \\
\text { dropping }\end{array}$ & $\begin{array}{l}\text { Secret key based mutual authentication } \\
\text { and symmetric key based encryption and } \\
\text { decryption }\end{array}$ & $\begin{array}{l}\text { Confidentiality, Authentica- } \\
\text { tion and Integrity }\end{array}$ \\
\hline [133] (2021) & $\begin{array}{l}\text { Multi-nodes multi- } \\
\text { hop UWASN }\end{array}$ & $\begin{array}{l}\text { Blackhole (also } \\
\text { known as packet- } \\
\text { dropping attack) }\end{array}$ & $\begin{array}{l}\text { Clustering with public key cryptography } \\
\text { and challenge response mechanism }\end{array}$ & $\begin{array}{l}\text { Authentication and Avail- } \\
\text { ability }\end{array}$ \\
\hline [94] (2021) & $\begin{array}{l}\text { Multi-nodes single- } \\
\text { hop UWASN }\end{array}$ & $\begin{array}{l}\text { Spoofing } \\
\text { (masquerade, sybil, } \\
\text { reply, time delay) } \\
\text { and Eavesdropping, }\end{array}$ & Symmetric-key cryptography & $\begin{array}{l}\text { Confidentiality and Authen- } \\
\text { tication }\end{array}$ \\
\hline [134] (2021) & $\begin{array}{l}\text { Multi-nodes single- } \\
\text { hop UWASN }\end{array}$ & Spoofing & $\begin{array}{l}\text { Machine learning based authentication: } \\
\text { channel taps, delay spread and received } \\
\text { power are exploited as input features for } \\
\text { neural network }\end{array}$ & Authentication \\
\hline [95] (2021) & Two legitimate nodes & $\begin{array}{l}\text { Spoofing and Eaves- } \\
\text { dropping }\end{array}$ & $\begin{array}{l}\text { Shared secret key generation: CIR based } \\
\text { four features extraction and quantization }\end{array}$ & $\begin{array}{l}\text { Confidentiality and Authen- } \\
\text { tication }\end{array}$ \\
\hline [96] (2021) & $\begin{array}{l}\text { Two legitimate and a } \\
\text { malicious nodes }\end{array}$ & $\begin{array}{l}\text { Spoofing and Eaves- } \\
\text { dropping }\end{array}$ & $\begin{array}{l}\text { Shared secret key generation: Information } \\
\text { Reconciliation and Privacy Amplification }\end{array}$ & $\begin{array}{l}\text { Confidentiality and Authen- } \\
\text { tication }\end{array}$ \\
\hline [135] (2021) & $\begin{array}{l}\text { Multi-nodes multi- } \\
\text { hop UWASN }\end{array}$ & Sybil & $\begin{array}{l}\text { Trust management model based on Hier- } \\
\text { archical Fuzzy System (HFS) }\end{array}$ & Authentication \\
\hline [136] (2022) & $\begin{array}{l}\text { Two legitimate and a } \\
\text { malicious nodes }\end{array}$ & Spoofing & $\begin{array}{l}\text { The estimated time-reversed CIR is con- } \\
\text { volved with the CIRs stored in a database }\end{array}$ & Authentication \\
\hline [137] (2022) & $\begin{array}{l}\text { Multi-nodes multi- } \\
\text { hop UWASN }\end{array}$ & $\begin{array}{l}\text { Spoofing } \\
\text { (specifically, depth- } \\
\text { spoofing attacks, } \\
\text { also known as sink- } \\
\text { hole attacks) }\end{array}$ & $\begin{array}{l}\text { Secure routing: energy-efficient depth- } \\
\text { based probabilistic routing protocol }\end{array}$ & $\begin{array}{l}\text { Authentication and Avail- } \\
\text { ability }\end{array}$ \\
\hline
\end{tabular}

TABLE VI: Summary of the reported security work in underwater acoustic communication systems (2021-till date).

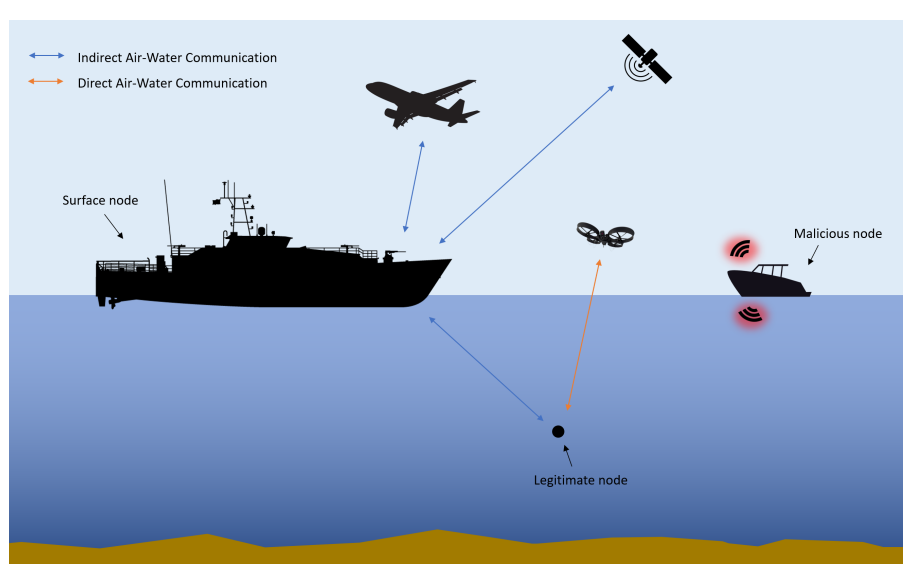

Fig. 9: Illustration of a scenario where a malicious node at the surface attacks the ongoing communication between underwater and airborne nodes. The malicious node can potentially lunch active and passive attacks in both mediums (however, such malicious node is not considered yet) or single medium affecting end-to-end communication. the security challenges of the indirect [107-110],[111] and direct [73, 112-114] A-W WCNs, which is not surprising given that this technology is still in its infancy. The authors in [107], for the first time, study the air-water communication with RF (in the air) and optical (in water) connections. They performed secrecy outage probability analysis using end-toend Signal to Noise Ratio (SNR) from the Low Earth Orbit (LEO) satellite to a submarine. Later, the authors in [111] studied physical layer confidentiality with an amplify-andforward relay node. Similarly, the authors in [108] provide physical layer confidentiality using decode-and-forward relay protocol at the surface node, while the authors in [109] exploit the multiple antennas at the decode-and-forward relay/surface node for physical layer confidentiality. Finally, authors in [110] provide secrecy outage probability expression using generalized Gamma fading at the RF link and mixture exponential generalized Gamma fading at the underwater optical link.

Moreover, recently some theoretical study on QKD considered direct air-water wireless communication using light in both mediums[73, 112-114]. The authors in [73] proposed CV-QKD for satellite to submarine communication, where submarine is considered at a depth of less than $100 \mathrm{~m}$. Similarly, the authors in [113], performed Monte-Carlo simulations 
for CV-QKD. More-recently, the authors in [114] used measurement device independent CV-QKD to improve the results (i.e., secret key generation) of previous studies.

\section{Conclusion, Gaps and Open Problems}

In this paper, we discussed the state-of-the-art mechanisms used for underwater and air-water wireless communication systems and further reported the existing security-related works in both mediums, mainly in terms of confidentiality, authentication and integrity. The three widely used technologies (RF, optics and acoustics) used in underwater and air-water wireless communication were discussed emphasizing the their security related issues. We can summarize our conclusions as follows:

- Despite the lack of interest in underwater RF communication in the past due to its limitations, some recent works have given breaths to this technology.

- The security in underwater RF communication has not been studied yet.

- In underwater wireless optical communication, the literature does not contain contributions on integrity and authentication (except few theoretical works, which studied only QKD in direct light air-water wireless communication); instead, the literature seems to be mainly focusing on confidentiality.

- In underwater acoustic communication, confidentiality, authentication and integrity were studied with various assumptions and system models.

- The literature does not contain studies on authentication and integrity in direct air-water wireless communication systems.

- Few recent works on the security of direct air-water wireless communication are all theoretical and consider one configuration of air-water wireless communication (i.e., light in both mediums).

- In air-water setting, no malicious node that is capable of simultaneously launching attacks in both mediums has been considered so far.

Despite the existing literature on the security of underwater and air-water wireless communication systems and previously mentioned research gaps, there are still many open problems, which we briefly discuss in the following subsections.

\section{A. Smart Malicious nodes}

A smart malicious node is a node that can assess the situation for launching attacks, for example a smart malicious node can be a node that can switch between passive and active modes on the fly $[185,186]$. Therefore, an interesting problem is to analyze the existing state-of-the-art underwater communication technologies under smart malicious nodes. In particular, it will be interesting to conduct such security analysis in the presence of malicious nodes equipped with the state-of-the-art machinery (i.e., Multiple Input and Multiple Output (MIMO), full duplex, GPUs capable of launching attacks in both mediums). The authors in a recent work [187] developed a full-duplex system using thermoacoustic effect and microwave vibration measurement for direct airwater wireless communication system using $10 \mathrm{~cm}$ dipped water node and $30 \mathrm{~cm}$ airborne node. This can naturally be extended to investigating security aspects of such communication. Additionally, to the best of our knowledge, a fullduplex malicious node is not considered in the literature. On the other hand, active attacks, such as jamming and spoofing, and passive attacks, such as eavesdropping, on direct airwater wireless communication are not explored yet. Hence, we believe that studying the security aspect of direct airwater wireless communication should be considered for future work. Similarly, we also need to account for malicious nodes capable of listening in both mediums in air-water wireless communication. In fact, this area lacks formal treatment of threat modelling to capture the malicious node's wide range of behaviors, strategies, the computing and communication facilities. The literature so far misses contributions on modeling of the malicious nodes themselves.

\section{B. Impact of Security mechanisms on other system parameters}

It is worth noting that adding security of a system will introduce an unavoidable overhead and implications. Since the overall performance of any system is a tangled mix of various system parameters, it is important to study the impact of security on relevant performance metrics, such as energy efficiency, throughput and reliability.

Indeed, some works discussed such trade-off between security and performance on wireless communications systems [188-194]. In [194], the authors provided relation of effective capacity and authentication parameters (false alarm and missed detection) as a closed-form expression in underwater acoustic communication, where they investigated the impact of physical layer authentication on effective capacity (a link layer metric that assesses the reliability level offered by a system).

Similarly, we believe it is necessary to thoroughly investigate the trade-off between security mechanisms and throughput, energy, spectral efficiencies of both underwater and airwater wireless communication systems in order to assess the cost of enforcing a security mechanism, given a desired level of security.

\section{AI/Machine Learning-empowered Security solutions}

Recently, machine learning tools have been leveraged to improving the performance of wireless communication networks [195-198]. From security perspective in the air-water communication systems, a recently reported work [87] used trained artificial neural networks to combat eavesdropping in underwater acoustic communication. Similarly, in underwater acoustic sensor networks, there has been an interest in using machine learning tools for, e.g., authentication [127, 134] and counter jamming [125]. As security improvements provided by machine learning tools in underwater communication looks quite promising, we believe that experimenting on air-water communication will most likely yields equally interesting results. 


\section{Cross-layer Security}

To further enhance the security and privacy of a communication network, cross-layer approaches have been used where security is provided at different protocol stack layers [199202]. Unlike the traditional security approaches where security is provided at a particular layer, cross layer approaches study security in multiple layers and protects information as it crosses the layers. We believe that such approaches have not been studied in air-water communication systems and can potentially improve the security in these systems.

\section{E. Secure Localization}

Localization continues to be an important topic for underwater nodes because of the unavailability of Global Positioning System (GPS) signals underwater. Instead, some reference/anchored nodes were used to localize deployed unknown underwater nodes [14, 203-206]. However, an open problem is to investigate localization of underwater nodes in direct air-water systems, possibly by complementing both reference/surface underwater nodes, and traditional localization mechanisms from over water. On the other hand, recently reported localization attacks, such as Sybil and Wormhole [207-209] imply that there is a great need to develop secure localization schemes. Some efforts have been made in this direction [18, 210-213], but more investigations are needed, particularly, for emerging communication technologies in airwater communication.

\section{ACKNOWLEDGEMENT}

This work is partially funded by the G5828 "SeaSec: DroNets for Maritime Border and Port Security" project under the NATO's Science for Peace Programme.

\section{REFERENCES}

[1] H. Kaushal and G. Kaddoum, "Underwater optical wireless communication," IEEE Access, vol. 4, pp. 1518-1547, 2016.

[2] Z. Zeng, S. Fu, H. Zhang, Y. Dong, and J. Cheng, "A survey of underwater optical wireless communications," IEEE Communications Surveys Tutorials, vol. 19, no. 1, pp. 204-238, 2017.

[3] C. Gussen, P. Diniz, M. Campos, W. Martins, F. Costa, and J. Gois, "A survey of underwater wireless communication technologies," Journal of Communication and Information Systems, vol. 31, no. 1, Oct. 2016.

[4] T. Khan, I. Ahmad, W. Aman, I. Azam, Z. A. Khan, U. Qasim, S. Avais, and N. Javaid, "Clustering depth based routing for underwater wireless sensor networks," in 2016 IEEE 30th International Conference on Advanced Information Networking and Applications (AINA), March 2016, pp. 506-515.

[5] "Extremely low frequency transmitter site clam lake, wisconsin," 2003. [Online]. Available: https://nuke.fas.org/guide/usa/c3i/fs-clamlake-elf2003.pdf

[6] A. Quazi and W. Konrad, "Underwater acoustic communications,' IEEE Communications Magazine, vol. 20, no. 2, pp. 24-30, 1982.

[7] F. Tonolini and F. Adib, "Networking across boundaries: Enabling wireless communication through the water-air interface," ser. SIGCOMM '18. New York, NY, USA: Association for Computing Machinery, 2018, p. 117â131.

[8] M. S. Islam, M. Younis, and A. Ahmed, "Communication through air water interface using multiple light sources," in 2018 IEEE International Conference on Communications (ICC), 2018, pp. 1-6.

[9] M. Kong, J. Wang, Y. Chen, T. Ali, R. Sarwar, Y. Qiu, S. Wang, J. Han, and J. Xu, "Security weaknesses of underwater wireless optical communication," Opt. Express, vol. 25, no. 18, pp. 21 509-21 518, Sep 2017.
[10] G. Han, J. Jiang, N. Sun, and L. Shu, "Secure communication for underwater acoustic sensor networks," IEEE communications magazine, vol. 53, no. 8, pp. 54-60, 2015.

[11] M. C. Domingo, "Securing underwater wireless communication networks," IEEE Wireless Communications, vol. 18, no. 1, pp. 22-28, 2011.

[12] P. Lacovara, "High-bandwidth underwater communications," Marine Technology Society Journal, vol. 42, no. 1, pp. 93-102, 2008.

[13] M.-A. Khalighi, C. Gabriel, T. Hamza, S. Bourennane, P. LÃ@on, and V. Rigaud, "Underwater wireless optical communication; recent advances and remaining challenges," in 2014 16th International Conference on Transparent Optical Networks (ICTON), 2014, pp. 1-4.

[14] N. Saeed, A. Celik, T. Y. Al-Naffouri, and M.-S. Alouini, "Underwater optical wireless communications, networking, and localization: A survey," Ad Hoc Networks, vol. 94, p. 101935, 2019.

[15] P. K. Sajmath, R. V. Ravi, and K. A. Majeed, "Underwater wireless optical communication systems: A survey," in 2020 7th International Conference on Smart Structures and Systems (ICSSS), 2020, pp. 1-7.

[16] K. Y. Islam, I. Ahmad, D. Habibi, and A. Waqar, "A survey on energy efficiency in underwater wireless communications," Journal of Network and Computer Applications, vol. 198, p. 103295, 2022.

[17] C. Lal, R. Petroccia, M. Conti, and J. Alves, "Secure underwater acoustic networks: Current and future research directions," in 2016 IEEE Third Underwater Communications and Networking Conference (UComms), 2016, pp. 1-5.

[18] G. Yang, L. Dai, G. Si, S. Wang, and S. Wang, "Challenges and security issues in underwater wireless sensor networks," Procedia Computer Science, vol. 147, pp. 210-216, 2019.

[19] S. Jiang, "On securing underwater acoustic networks: A survey," IEEE Communications Surveys \& Tutorials, vol. 21, no. 1, pp. 729-752, 2018.

[20] https://www.popotomodem.com/.

[21] http://www.aquasent.com/acoustic-modems/.

[22] https://www.hydromea.com/underwater-wireless-communication.

[23] R. K. Moore, "Radio communication in the sea," IEEE Spectrum, vol. 4, no. 11, pp. 42-51, 1967.

[24] M. Siegel and R. King, "Electromagnetic propagation between antennas submerged in the ocean," IEEE Transactions on Antennas and Propagation, vol. 21, no. 4, pp. 507-513, 1973.

[25] D. Pompili and I. F. Akyildiz, "Overview of networking protocols for underwater wireless communications," IEEE Communications Magazine, vol. 47, no. 1, pp. 97-102, 2009.

[26] X. Che, I. Wells, G. Dickers, P. Kear, and X. Gong, "Re-evaluation of rf electromagnetic communication in underwater sensor networks," IEEE Communications Magazine, vol. 48, no. 12, pp. 143-151, 2010.

[27] A. Palmeiro, M. Mart ̃̂n, I. Crowther, and M. Rhodes, "Underwater radio frequency communications," in OCEANS 2011 IEEE - Spain, 2011, pp. 1-8.

[28] S. Jiang and S. Georgakopoulos, "Electromagnetic wave propagation into fresh water," Journal of Electromagnetic Analysis and Applications, vol. 2011, 2011.

[29] U. M. Qureshi, F. K. Shaikh, Z. Aziz, S. M. Z. S. Shah, A. A. Sheikh, E. Felemban, and S. B. Qaisar, "Rf path and absorption loss estimation for underwater wireless sensor networks in different water environments," Sensors, vol. 16, no. 6, p. 890, 2016.

[30] P. Saini, R. P. Singh, and A. Sinha, "Path loss analysis of rf waves for underwater wireless sensor networks," in 2017 International Conference on Computing and Communication Technologies for Smart Nation (IC3TSN), 2017, pp. 104-108.

[31] M. Soomro, S. N. Azar, O. Gurbuz, and A. Onat, "Work-in-progress: Networked control of autonomous underwater vehicles with acoustic and radio frequency hybrid communication," in 2017 IEEE Real-Time Systems Symposium (RTSS), 2017, pp. 366-368.

[32] K. G. Omeke, A. Abohmra, M. A. Imran, Q. H. Abbasi, and L. Zhang, "Characterization of rf signals in different types of water," in Antennas and Propagation Conference 2019 (APC-2019), 2019, pp. 1-6.

[33] S. M. Maher, Z. M. Ali, H. H. Mahmoud, S. O. Abdellatif, and M. M. Abdellatif, "Performance of rf underwater communications operating at $433 \mathrm{mhz}$ and $2.4 \mathrm{ghz}$," in 2019 International Conference on Innovative Trends in Computer Engineering (ITCE), 2019, pp. 334339.

[34] P. P. Ganesh and H. Venkataraman, "Rf-based multihop wireless communication for shallow underwater environment," in 2019 International Conference on Wireless Communications Signal Processing 
and Networking (WiSPNET), 2019, pp. 222-228.

[35] M. Ramdhan, M. Ali, S. Ali, M. Kamaludin, et al., "Measuring the underwater received power behavior for $433 \mathrm{mhz}$ radio frequency based on different distance and depth for the development of an underwater wireless sensor network," Bulletin of Electrical Engineering and Informatics, vol. 8, no. 3, pp. 1066-1073, 2019.

[36] K. Takizawa, R. Suga, T. Matsuda, and F. Kojima, "Underwater mimo communications by rf signals: Capacity analysis, simulations, and experiment," in 2021 Joint European Conference on Networks and Communications 6G Summit (EuCNC/6G Summit), 2021, pp. 460-465.

[37] A. Zoksimovski, D. Sexton, M. Stojanovic, and C. Rappaport, "Underwater electromagnetic communications using conduction-channel characterization," Ad Hoc Networks, vol. 34, pp. 42-51, 2015.

[38] I. F. Akyildiz, D. Pompili, and T. Melodia, "Underwater acoustic sensor networks: research challenges," Ad Hoc Networks, vol. 3, no. 3, pp. 257-279, 2005

[39] S. Jiang, "On reliable data transfer in underwater acoustic networks: A survey from networking perspective," IEEE Communications Surveys Tutorials, vol. 20, no. 2, pp. 1036-1055, 2018.

[40] W. Zhang, M. Stojanovic, and U. Mitra, "Analysis of a linear multihop underwater acoustic network," IEEE Journal of Oceanic Engineering, vol. 35 , no. 4, pp. 961-970, 2010.

[41] D. R. Andrews, "Ultrasonics and acoustics," in Encyclopedia of Physical Science and Technology (Third Edition), third edition ed., R. A. Meyers, Ed. New York: Academic Press, 2003, pp. 269-287.

[42] S. Al-Dharrab, M. Uysal, and T. M. Duman, "Cooperative underwater acoustic communications [accepted from open call]," IEEE Communications Magazine, vol. 51, no. 7, pp. 146-153, 2013.

[43] T. Bian, R. Venkatesan, and C. Li, "Design and evaluation of a new localization scheme for underwater acoustic sensor networks," in GLOBECOM 2009 - 2009 IEEE Global Telecommunications Conference, 2009, pp. 1-5.

[44] M. Stojanovic, "On the relationship between capacity and distance in an underwater acoustic communication channel," in Proceedings of the 1st ACM International Workshop on Underwater Networks, ser. WUWNet '06. New York, NY, USA: ACM, 2006, pp. 41-47.

[45] _ - "On the relationship between capacity and distance in an underwater acoustic communication channel," SIGMOBILE Mob. Comput. Commun. Rev., vol. 11 , no. 4, p. 34â43, Oct. 2007.

[46] S. Karp, "Optical communications between underwater and above surface (satellite) terminals," IEEE Transactions on Communications, vol. 24 , no. 1 , pp. $66-81,1976$.

[47] H. M. Oubei, C. Shen, A. Kammoun, E. Zedini, K.-H. Park, X. Sun, G. Liu, C. H. Kang, T. K. Ng, M.-S. Alouini, and B. S. Ooi, "Light based underwater wireless communications," vol. 57, no. 8S2, p. 08PA06, jul 2018.

[48] X. Huang, F. Yang, and J. Song, "Hybrid ld and led-based underwater optical communication: state-of-the-art, opportunities, challenges, and trends," Chin. Opt. Lett., vol. 17, no. 10, p. 100002, Oct 2019.

[49] Q. Yan, Z. Li, Z. Hong, T. Zhan, and Y. Wang, "Photon-counting underwater wireless optical communication by recovering clock and data from discrete single photon pulses," IEEE Photonics Journal, vol. 11, no. 5, pp. 1-15, 2019.

[50] G. Schirripa Spagnolo, L. Cozzella, and F. Leccese, "Underwater optical wireless communications: Overview," Sensors, vol. 20, no. 8 , 2020

[51] S. Zhu, X. Chen, X. Liu, G. Zhang, and P. Tian, "Recent progress in and perspectives of underwater wireless optical communication,' Progress in Quantum Electronics, vol. 73, p. 100274, 2020.

[52] X. Sun, C. H. Kang, M. Kong, O. Alkhazragi, Y. Guo, M. Ouhssain, Y. Weng, B. H. Jones, T. K. Ng, and B. S. Ooi, "A review on practical considerations and solutions in underwater wireless optical communication," J. Lightwave Technol., vol. 38, no. 2, pp. 421-431, Jan 2020.

[53] M. Sait, Y. Guo, O. Alkhazragi, M. Kong, T. K. Ng, and B. S Ooi, "The impact of vertical salinity gradient on non-line-of-sight underwater optical wireless communication," IEEE Photonics Journal, vol. 13 , no. 6 , pp. 1-9, 2021.

[54] I. U. Khan, B. Iqbal, L. Songzou, H. Li, G. Qiao, and S. Khan, "Full-duplex underwater optical communication systems: A review," in 2021 International Bhurban Conference on Applied Sciences and Technologies (IBCAST), 2021, pp. 886-893.

[55] H. Lu, M. Jiang, and J. Cheng, "Deep learning aided robust joint channel classification, channel estimation, and signal detection for underwater optical communication," IEEE Transactions on Communications, vol. 69, no. 4, pp. 2290-2303, 2021

[56] F. Akhoundi, J. A. Salehi, and A. Tashakori, "Cellular underwater wireless optical cdma network: Performance analysis and implementation concepts," IEEE Transactions on Communications, vol. 63, no. 3, pp. $882-891,2015$.

[57] S. Tang, Y. Dong, and X. Zhang, "Impulse response modeling for underwater wireless optical communication links," IEEE Transactions on Communications, vol. 62, no. 1, pp. 226-234, 2014.

[58] Z. Ji, Y. Fu, J. Li, Z. Zhao, and W. Mai, "Photoacoustic communication from the air to underwater based on low-cost passive relays," IEEE Communications Magazine, vol. 59, no. 1, pp. 140-143, 2021.

[59] S. Anees and R. Deka, "On the performance of df based dual-hop mixed rf amp;x002f;uwoc system," in 2019 IEEE 89th Vehicular Technology Conference (VTC2019-Spring), 2019, pp. 1-5.

[60] C. J. Carver, Z. Tian, H. Zhang, K. M. Odame, A. Quattrini Li, and X. Zhou, "Amphilight: Direct air-water communication with laser light," GetMobile: Mobile Comp. and Comm., vol. 24, no. 3, p. 26â29, Jan. 2021.

[61] M. S. Islam and M. F. Younis, "Analyzing visible light communication through airâwater interface," IEEE Access, vol. 7, pp. 123 830-123 845, 2019.

[62] P. Nabavi, A. F. M. S. Haq, and M. Yuksel, "Empirical modeling and analysis of water-to-air optical wireless communication channels," in 2019 IEEE International Conference on Communications Workshops (ICC Workshops), 2019, pp. 1-6.

[63] L.-K. Chen, Y. Shao, and Y. Di, "Underwater and water-air optical wireless communication," in 2021 Optical Fiber Communications Conference and Exhibition (OFC), 2021, pp. 1-3.

[64] T. Lin, N. Huang, C. Gong, J. Luo, and Z. Xu, "Preliminary characterization of coverage for water-to-air visible light communication through wavy water surface," IEEE Photonics Journal, vol. 13, no. 1, pp. 1-13, 2021.

[65] Y. Shao, Y. Di, and L.-K. Chen, "Adaptive loading for water-air simo owc system based on the temporal and spatial properties of waves," in 2021 Optical Fiber Communications Conference and Exhibition $(O F C), 2021$, pp. 1-3.

[66] X. Sun, M. Kong, C. Shen, C. H. Kang, T. K. Ng, and B. S. Ooi, “On the realization of across wavy water-air-interface diffuse-line-of-sight communication based on an ultraviolet emitter," Opt. Express, vol. 27, no. 14, pp. 19635-19649, Jul 2019.

[67] X. Sun, M. Kong, O. A. Alkhazragi, K. Telegenov, M. Ouhssain, M. Sait, Y. Guo, B. H. Jones, J. S. Shamma, T. K. Ng, and B. S. Ooi, "Field demonstrations of wide-beam optical communications through waterâair interface," IEEE Access, vol. 8, pp. 160480-160 489, 2020.

[68] K. Enhos, E. Demirors, D. Unal, and T. Melodia, "Software-defined visible light networking for bi-directional wireless communication across the air-water interface," in 2021 18th Annual IEEE International Conference on Sensing, Communication, and Networking (SECON), 2021, pp. 1-9.

[69] M. Watson, J.-F. Bousquet, and A. Forget, "Evaluating the feasibility of magnetic induction to cross an air-water boundary," International Journal of Electronics and Communication Engineering, vol. 15, no. 6, pp. $262-265,2021$

[70] C. Guo, B. Deng, Q. Yang, H. Wang, and K. Liu, "Modeling and simulation of water-surface vibration due to acoustic signals for detection with terahertz radar," in 2019 12th UK-Europe-China Workshop on Millimeter Waves and Terahertz. Technologies (UCMMT), 2019, pp. $1-3$.

[71] M. R. Romero, R. M. Narayanan, E. H. Lenzing, D. C. Brown, and K. L. Greenert, "Wireless underwater-to-air communications via water surface modulation and radar detection," in Radar Sensor Technology XXIV, K. I. Ranney and A. M. Raynal, Eds., vol. 11408, International Society for Optics and Photonics. SPIE, 2020, pp. $58-71$.

[72] Z. Luo, R. Guo, and M. Liu, "Parameter conditions for phase unwrapping and coherent processing in the tarf communication," in 2019 IEEE 5th International Conference on Computer and Communications (ICCC), 2019, pp. 831-836.

[73] Y. Guo, C. Xie, P. Huang, J. Li, L. Zhang, D. Huang, and G. Zeng, "Channel-parameter estimation for satellite-to-submarine continuousvariable quantum key distribution," Physical Review A, vol. 97, no. 5, p. 052326,2018

[74] Y. Liu, J. Jing, and J. Yang, "Secure underwater acoustic communication based on a robust key generation scheme," in 2008 9th 
International Conference on Signal Processing, Oct 2008, pp. 1838 1841.

[75] G. Dini and A. L. Duca, "A cryptographic suite for underwater cooperative applications," in 2011 IEEE Symposium on Computers and Communications (ISCC), June 2011, pp. 870-875.

[76] G. Ateniese, A. Capossele, P. Gjanci, C. Petrioli, and D. Spaccini, "SecFUN: Security framework for underwater acoustic sensor networks," in OCEANS 2015 - Genova, May 2015, pp. 1-9.

[77] Q. Wang, H.-N. Dai, X. Li, H. Wang, and H. Xiao, "On modeling eavesdropping attacks in underwater acoustic sensor networks," Sensors, vol. 16, no. 5, 2016.

[78] Y. Huang, P. Xiao, S. Zhou, and Z. Shi, "A half-duplex self-protection jamming approach for improving secrecy of block transmissions in underwater acoustic channels," IEEE Sensors Journal, vol. 16, no. 11, pp. 4100-4109, June 2016.

[79] Y. Huang, S. Zhou, Z. Shi, and L. Lai, "Channel frequency responsebased secret key generation in underwater acoustic systems," IEEE Transactions on Wireless Communications, vol. 15, no. 9, pp. 58755888, Sept 2016.

[80] M. Xu and L. Liu, "Sensevault: A three-tier framework for securing mobile underwater sensor networks," IEEE Transactions on Mobile Computing, pp. 1-1, 2018.

[81] K. Pelekanakis, C. M. Gussen, R. Petroccia, and J. Alves, "Toward physical layer cryptography for underwater acoustic networking," in Proc. of the 5th International Underwater Acoustics Conference and Exhibition, 2019.

[82] — , "Robust channel parameters for crypto key generation in underwater acoustic systems," in OCEANS 2019 MTS/IEEE SEATTLE. IEEE, 2019, pp. 1-7.

[83] S. Bagali and R. Sundaraguru, "Maximize resource utilization based channel access model with presence of reactive jammer for underwater wireless sensor network," International Journal of Electrical and Computer Engineering, vol. 10, no. 3, p. 3284, 2020.

[84] _ "Efficient resource allocation design for mitigating multi-jammer in underwater wireless sensor network," EAI Endorsed Transactions on Internet of Things, vol. 6, no. 24, p. e2, 2020.

[85] F. Chiariotti, A. Signori, F. Campagnaro, and M. Zorzi, "Underwater jamming attacks as incomplete information games," in IEEE INFOCOM 2020-IEEE Conference on Computer Communications Workshops (INFOCOM WKSHPS). IEEE, 2020, pp. 1033-1038.

[86] K. Liang, H. Huang, X. Huang, and Q. Yang, "Cs-based homomorphism encryption and trust scheme for underwater acoustic sensor networks," in International Conference on Machine Learning and Big Data Analytics for IoT Security and Privacy. Springer, 2020, pp. 394-399.

[87] W. Aman, M. M. U. Rahman, Z. Haider, J. Qadir, M. W. Nawaz, and G. A. S. Sidhu, "Maximizing secrecy rate of an orthogonal frequency division multiplexing-based multihop underwater acoustic sensor network," Transactions on Emerging Telecommunications Technologies, vol. 31, no. 11 , p. e4106, 2020

[88] M. Xu, Y. Fan, and L. Liu, "Multi-party secret key generation over underwater acoustic channels," IEEE Wireless Communications Letters, vol. 9, no. 7, pp. 1075-1079, 2020.

[89] A. Signori, F. Chiariotti, F. Campagnaro, and M. Zorzi, "A gametheoretic and experimental analysis of energy-depleting underwate jamming attacks," IEEE Internet of Things Journal, vol. 7, no. 10 , pp. 9793-9804, 2020.

[90] A. Ozmen, H. U. Yildiz, and B. Tavli, "Impact of minimizing the eavesdropping risks on lifetime of underwater acoustic sensor networks," in 2020 28th Telecommunications Forum (TELFOR). IEEE, 2020, pp. $1-4$.

[91] X. Li, Y. Zhou, L. Yan, H. Zhao, X. Yan, and X. Luo, "Optimal node selection for hybrid attack in underwater acoustic sensor networks: A virtual expert-guided bandit algorithm," IEEE Sensors Journal, vol. 20, no. 3, pp. 1679-1687, 2020.

[92] A. Signori, F. Chiariotti, F. Campagnaro, R. Petroccia, K. Pelekanakis, P. Paglierani, J. Alves, and M. Zorzi, "A geometry-based game theoretical model of blind and reactive underwater jamming," IEEE Transactions on Wireless Communications, 2021.

[93] X. Yu, H. Chen, and L. Xie, "A secure communication protocol between sensor nodes and sink node in underwater acoustic sensor networks," in 2021 IEEE International Conference on Artificial Intelligence and Computer Applications (ICAICA), 2021, pp. 279-283.

[94] U. Jain and M. Hussain, "Security mechanism for maritime territory and frontier surveillance in naval operations using wireless senso networks," Concurrency and Computation: Practice and Experience, vol. 33, no. 17, p. e6300, 2021

[95] K. Pelekanakis, S. A. Yıldırım, G. Sklivanitis, R. Petroccia, J. Alves, and D. Pados, "Physical layer security against an informed eavesdropper in underwater acoustic channels: Feature extraction and quantization," in 2021 Fifth Underwater Communications and Networking Conference (UComms). IEEE, pp. 1-5.

[96] G. Sklivanitis, K. Pelekanakis, S. A. Yıldırım, R. Petroccia, J. Alves, and D. A. Pados, "Physical layer security against an informed eavesdropper in underwater acoustic channels: Reconciliation and privacy amplification," in 2021 Fifth Underwater Communications and Networking Conference (UComms). IEEE, pp. 1-5.

[97] G. D. Verma, A. Mathur, Y. Ai, and M. Cheffena, "Secrecy performance of fso communication systems with non-zero boresight pointing errors," IET Communications, vol. 15, no. 1, pp. 155-162, 2021.

[98] D. Shaboy, D. Rockban, and A. Handelman, "Tapping underwater wireless optical communication in pure water and natural dead-sea ultra-high-salinity water by diffraction grating," Opt. Express, vol. 26 , no. 23, pp. 29700-29711, Nov 2018.

[99] J. Du, Y. Wang, C. Fei, R. Chen, G. Zhang, X. Hong, and S. He, "Experimental demonstration of 50-m/5-gbps underwater optical wireless communication with low-complexity chaotic encryption," Opt Express, vol. 29, no. 2, pp. 783-796, Jan 2021.

[100] P. Shi, S.-C. Zhao, Y.-J. Gu, and W.-D. Li, "Channel analysis for single photon underwater free space quantum key distribution," JOSA $A$, vol. 32, no. 3, pp. 349-356, 2015.

[101] Q. Peng, G. Chen, X. Li, Q. Liao, and Y. Guo, "Performance improvement of underwater continuous-variable quantum key distribution via photon subtraction," Entropy, vol. 21, no. 10, p. 1011, 2019.

[102] J. Gariano and I. B. Djordjevic, "Theoretical study of a submarine to submarine quantum key distribution systems," Optics express, vol. 27, no. 3, pp. 3055-3064, 2019.

[103] Y. Mao, X. Wu, W. Huang, Q. Liao, H. Deng, Y. Wang, and Y. Guo, "Monte carlo-based performance analysis for underwater continuousvariable quantum key distribution," Applied Sciences, vol. 10, no. 17, p. $5744,2020$.

[104] Y. Wang, S. Zou, Y. Mao, and Y. Guo, "Improving underwater continuous-variable measurement-device-independent quantum key distribution via zero-photon catalysis," Entropy, vol. 22, no. 5, p. 571, 2020

[105] Y. Xiang, Y. Wang, X. Ruan, Z. Zuo, and Y. Guo, "Improving the discretely modulated underwater continuous-variable quantum key distribution with heralded hybrid linear amplifier," Physica Scripta vol. 96, no. 6, p. 065103, 2021.

[106] Z. Zuo, Y. Wang, Y. Mao, X. Ruan, and Y. Guo, "Security of quantum communications in oceanic turbulence," Physical Review A, vol. 104, no. 5 , p. 052613,2021

[107] E. Illi, F. El Bouanani, D. B. Da Costa, F. Ayoub, and U. S Dias, "Dual-hop mixed rf-uow communication system: A phy security analysis," IEEE Access, vol. 6, pp. 55 345-55 360, 2018.

[108] Y. Lou, R. Sun, J. Cheng, D. Nie, and G. Qiao, "Secrecy outage analysis of two-hop decode-and-forward mixed rf/uwoc systems," IEEE Communications Letters, pp. 1-1, 2021.

[109] E. Illi, F. E. Bouanani, D. B. da Costa, P. C. Sofotasios, F. Ayoub, K. Mezher, and S. Muhaidat, "Physical layer security of a dual-hop regenerative mixed rf/uow system," IEEE Transactions on Sustainable Computing, vol. 6, no. 1, pp. 90-104, 2021.

[110] A. S. M. Badrudduza, M. Ibrahim, S. M. R. Islam, M. S. Hossen, M. K. Kundu, I. S. Ansari, and H. Yu, "Security at the physical layer over gg fading and megg turbulence induced rf-uowc mixed system," IEEE Access, vol. 9, pp. 18 123-18 136, 2021.

[111] Y. Lou, R. Sun, J. Cheng, S. Liu, F. Zhou, and G. Qiao, "Physicallayer security for two-hop air-to-underwater communication systems with fixed-gain amplify-and-forward relaying," 2020.

[112] H.-b. Xu, Y.-y. Zhou, X.-j. Zhou, and L. Wang, "Performance analysis of air-water quantum key distribution with an irregular sea surface," Optoelectronics Letters, vol. 14, no. 3, pp. 216-219, 2018.

[113] C.-L. Xie, Y. Guo, Y.-J. Wang, D. Huang, and L. Zhang, "Security simulation of continuous-variable quantum key distribution over airto-water channel using monte carlo method," Chinese Physics Letters, vol. 35, no. 9, p. 090302, 2018.

[114] Q. Peng, Y. Guo, Q. Liao, and X. Ruan, "Satellite-to-submarine quantum communication based on measurement-device-independent 
continuous-variable quantum key distribution," Quantum Information Processing, vol. 21, no. 2, pp. 1-19, 2022.

[115] G. Dini and A. L. Duca, "Seflood: A secure network discovery protocol for underwater acoustic networks," in 2011 IEEE Symposium on Computers and Communications (ISCC), June 2011, pp. 636-638.

[116] R. Diamant, P. Casari, and S. Tomasin, "Cooperative authentication in underwater acoustic sensor networks," IEEE Transactions on Wireless Communications, vol. 18, no. 2, pp. 954-968, 2019.

[117] F. Campagnaro, D. Tronchin, A. Signori, R. Petroccia, K. Pelekanakis, P. Paglierani, J. Alves, and M. Zorzi, "Replay-attack countermeasures for underwater acoustic networks," in Global Oceans 2020: SingaporeUS Gulf Coast. IEEE, 2020, pp. 1-9.

[118] R. Zhang and Y. Zhang, "Wormhole-resilient secure neighbor discovery in underwater acoustic networks," in 2010 Proceedings IEEE INFOCOM, March 2010, pp. 1-9.

[119] E. Souza, H. C. Wong, I. Cunha, Ã. Cunha, L. F. M. Vieira, and L. B. Oliveira, "End-to-end authentication in under-water sensor networks," in 2013 IEEE Symposium on Computers and Communications (ISCC), 2013, pp. 000 299-000304.

[120] X. Li, G. Han, A. Qian, L. Shu, and J. Rodrigues, "Detecting sybil attack based on state information in underwater wireless sensor networks," in 2013 21st International Conference on Software, Telecommunications and Computer Networks - (SoftCOM 2013), 2013, pp. 1-5.

[121] Y. Li, L. Xiao, Q. Li, and W. Su, "Spoofing detection games in underwater sensor networks," in OCEANS 2015-MTS/IEEE Washington. IEEE, 2015, pp. 1-5.

[122] X. Du, C. Peng, and K. Li, "A secure routing scheme for underwater acoustic networks," International Journal of Distributed Sensor Networks, vol. 13, no. 6, p. 1550147717713643, 2017.

[123] Z. Liu, X. Deng, and J. Li, "A secure localization algorithm based on reputation against wormhole attack in uwsns," in 2017 International Symposium on Intelligent Signal Processing and Communication Systems (ISPACS). IEEE, 2017, pp. 695-700.

[124] M. R. Bharamagoudra and S. S. Manvi, "Agent-based secure routing for underwater acoustic sensor networks," International Journal of Communication Systems, vol. 30, no. 13, p. e3281, 2017, e3281 dac. 3281

[125] L. Xiao, X. Wan, W. Su, Y. Tang, et al., "Anti-jamming underwater transmission with mobility and learning," IEEE Communications Letters, vol. 22, no. 3, pp. 542-545, 2018.

[126] W. Aman, M. M. U. Rahman, J. Qadir, H. Pervaiz, and Q. Ni, "Impersonation detection in line-of-sight underwater acoustic sensor networks," IEEE Access, vol. 6, pp. 44459-44472, Aug. 2018

[127] L. Xiao, G. Sheng, X. Wan, W. Su, and P. Cheng, "Learningbased phy-layer authentication for underwater sensor networks," IEEE Communications Letters, vol. 23, no. 1, pp. 60-63, 2019.

[128] K. Saeed, W. Khalil, S. Ahmed, I. Ahmad, and M. N. K. Khattak, "Seecr: Secure energy efficient and cooperative routing protocol for underwater wireless sensor networks," IEEE Access, vol. 8, pp. 107419 107433,2020

[129] M. Khalid, R. Zhao, and N. Ahmed, "Physical layer authentication in line-of-sight underwater acoustic sensor networks," in Global Oceans 2020: Singapore-US Gulf Coast. IEEE, 2020, pp. 1-5.

[130] M. Khalid, R. Zhao, and X. Wang, "Node authentication in underwater acoustic sensor networks using time-reversal," in Global Oceans 2020: Singapore-US Gulf Coast. IEEE, 2020, pp. 1-4.

[131] A. P. Das, S. M. Thampi, and J. Lloret, "Anomaly detection in uasn localization based on time series analysis and fuzzy logic," Mobile Networks and Applications, vol. 25, no. 1, pp. 55-67, 2020.

[132] X. Pan, Y. Shen, and J. Zhang, "Iout based underwater target localization in the presence of time synchronization attacks," IEEE Transactions on Wireless Communications, vol. 20, no. 6, pp. 39583973, 2021.

[133] D. Zala, D. Thummar, and B. R. Chandavarkar, "Mitigating blackhole attack of underwater sensor networks," in 2021 12th International Conference on Computing Communication and Networking Technologies (ICCCNT), 2021, pp. 1-8.

[134] L. Bragagnolo, F. Ardizzon, N. Laurenti, P. Casari, R. Diamant, and $\mathrm{S}$. Tomasin, "Authentication of underwater acoustic transmissions via machine learning techniques," in 2021 IEEE International Conference on Microwaves, Antennas, Communications and Electronic Systems (COMCAS), 2021, pp. 255-260.

[135] A. A. Islam and K. A. Taher, "A novel authentication mechanism for securing underwater wireless sensors from sybil attack," in 20215 th International Conference on Electrical Engineering and Information Communication Technology (ICEEICT), 2021, pp. 1-6.

[136] R. Zhao, M. Khalid, O. A. Dobre, and X. Wang, "Physical layer node authentication in underwater acoustic sensor networks using timereversal," IEEE Sensors Journal, pp. 1-1, 2022.

[137] A. Alharbi, A. M. Abbas, and S. Ibrahim, "Securing localization-free underwater routing protocols against depth-spoofing attacks," Array, vol. 13, p. 100117, 2022.

[138] M. Goetz, S. Azad, P. Casari, I. Nissen, and M. Zorzi, "Jammingresistant multi-path routing for reliable intruder detection in underwater networks," in Proceedings of the Sixth ACM International Workshop on Underwater Networks. ACM, 2011, p. 10

[139] S. Misra, "Jamming in underwater sensor networks: detection and mitigation," IET Communications, vol. 6, pp. 2178-2188(10), September 2012

[140] M. Zuba, Z. Shi, Z. Peng, J.-H. Cui, and S. Zhou, "Vulnerabilities of underwater acoustic networks to denial-of-service jamming attacks," Security and Communication Networks, vol. 8, no. 16, pp. 2635-2645, 2015

[141] L. Xiao, Q. Li, T. Chen, E. Cheng, and H. Dai, "Jamming games in underwater sensor networks with reinforcement learning," in 2015 IEEE Global Communications Conference (GLOBECOM), Dec 2015, pp. 1-6.

[142] S. Bagali and R. Sundaraguru, "Efficient channel access model for detecting reactive jamming for underwater wireless sensor network," in 2019 International Conference on Wireless Communications Signal Processing and Networking (WiSPNET). IEEE, 2019, pp. 196-200.

[143] R. L. Rivest, A. Shamir, and L. Adleman, "A method for obtaining digital signatures and public-key cryptosystems," Communications of the ACM, vol. 21, no. 2, pp. 120-126, 1978 .

[144] W. Diffie and M. Hellman, "New directions in cryptography," IEEE transactions on Information Theory, vol. 22, no. 6, pp. 644-654, 1976.

[145] P. W. Shor, "Algorithms for quantum computation: discrete logarithms and factoring," in Proceedings 35th annual symposium on foundations of computer science. Ieee, 1994, pp. 124-134.

[146] W. A. Arbaugh, N. Shankar, Y. J. Wan, and K. Zhang, "Your 80211 wireless network has no clothes," IEEE Wireless Communications, vol. 9, no. 6, pp. 44-51, 2002.

[147] C. Gidney and M. EkerÃ $¥$, "How to factor 2048 bit rsa integers in 8 hours using 20 million noisy qubits," 2019.

[148] Z.-C. Duan, J.-P. Li, J. Qin, Y. Yu, Y.-H. Huo, S. Höfling, C.-Y. Lu, N.L. Liu, K. Chen, and J.-W. Pan, "Proof-of-principle demonstration of compiled shorâs algorithm using a quantum dot single-photon source," Optics Express, vol. 28, no. 13, pp. 18917-18930, 2020.

[149] Y.-S. Shiu, S. Y. Chang, H.-C. Wu, S. C.-H. Huang, and H.-H. Chen, "Physical layer security in wireless networks: A tutorial," IEEE wireless Communications, vol. 18, no. 2, pp. 66-74, 2011.

[150] M. Bloch and J. Barros, Physical-layer security: from information theory to security engineering. Cambridge University Press, 2011.

[151] X. Zhou, L. Song, and Y. Zhang, Physical layer security in wireless communications. Crc Press, 2013

[152] W. Aman, G. A. S. Sidhu, T. Jabeen, F. Gao, and S. Jin, "Enhancing physical layer security in dual-hop multiuser transmission," in 2016 IEEE Wireless Communications and Networking Conference, April 2016, pp. 1-6.

[153] A. Mahmood, W. Aman, M. O. Iqbal, M. M. U. Rahman, and Q. H. Abbasi, "Channel impulse response-based distributed physical layer authentication," in 2017 IEEE 85th Vehicular Technology Conference (VTC Spring). IEEE, 2017, pp. 1-5.

[154] W. Aman, G. A. S. Sidhu, H. M. Furqan, and Z. Ali, "Enhancing physical layer security in af relay-assisted multicarrier wireless transmission," Transactions on Emerging Telecommunications Technologies, vol. 29, no. 6, p. e3289, 2018, e3289 ett.3289.

[155] W. Aman, A. Ijaz, M. M. U. Rahman, D. N. K. Jayakody, and H. Pervaiz, "Shared secret key generation via carrier frequency offsets," in 2019 IEEE 89th Vehicular Technology Conference (VTC2019-Spring). IEEE, 2019, pp. 1-5.

[156] S. Zafar, W. Aman, M. M. U. Rahman, A. Alomainy, and Q. H. Abbasi, "Channel impulse response-based physical layer authentication in a diffusion-based molecular communication system," in 2019 UK/China Emerging Technologies (UCET). IEEE, 2019, pp. 1-2.

[157] A. Mehmood, W. Aman, M. M. U. Rahman, M. A. Imran, and Q. H. Abbasi, "Preventing identity attacks in rfid backscatter communication 
systems: A physical-layer approach," in 2020 International Conference on UK-China Emerging Technologies (UCET), 2020, pp. 1-5.

[158] W. Aman, M. M. U. Rahman, H. T. Abbas, M. A. Khalid, M. A. Imran, A. Alomainy, and Q. H. Abbasi, "Securing the insecure: A first-line-ofdefense for body-centric nanoscale communication systems operating in thz band," Sensors, vol. 21, no. 10, p. 3534, 2021.

[159] A. D. Wyner, "The wire-tap channel," The Bell System Technical Journal, vol. 54, no. 8, pp. 1355-1387, Oct 1975.

[160] Z. Gao, H. Hu, D. Cheng, J. Xu, and X. Sun, "Physical layer security based on artificial noise and spatial modulation," in 2016 8th Inter national Conference on Wireless Communications Signal Processing (WCSP), 2016, pp. 1-5.

[161] M. Bloch and J. Barros, Physical-layer security: from information theory to security engineering. Cambridge University Press, 2011.

[162] L. Bai, L. Zhu, J. Liu, J. Choi, and W. Zhang, "Physical layer authentication in wireless communication networks: A survey," Journal of Communications and Information Networks, vol. 5, no. 3, pp. 237264, 2020.

[163] L. Xiao, L. J. Greenstein, N. B. Mandayam, and W. Trappe, "Using the physical layer for wireless authentication in time-variant channels," IEEE Transactions on Wireless Communications, vol. 7, no. 7, pp 2571-2579, 2008.

[164] J. Yang, Y. Chen, W. Trappe, and J. Cheng, "Detection and localization of multiple spoofing attackers in wireless networks," IEEE Transactions on Parallel and Distributed Systems, vol. 24, no. 1, pp. 44-58, 2013.

[165] P. Hao, X. Wang, and A. Behnad, "Performance enhancement of i/q imbalance based wireless device authentication through collaboration of multiple receivers," in 2014 IEEE International Conference on Communications (ICC), 2014, pp. 939-944.

[166] M. M. U. Rahman, A. Yasmeen, and Q. H. Abbasi, "Exploiting lack of hardware reciprocity for sender-node authentication at the phy layer," in 2017 IEEE 85th Vehicular Technology Conference (VTC Spring). IEEE, 2017, pp. 1-5.

[167] M. M. U. Rahman, A. Yasmeen, and J. Gross, "PHY layer authentication via drifting oscillators," in 2014 IEEE Global Communications Conference, Dec 2014, pp. 716-721.

[168] P. Baracca, N. Laurenti, and S. Tomasin, "Physical layer authentication over mimo fading wiretap channels," IEEE Transactions on Wireless Communications, vol. 11, no. 7, pp. 2564-2573, 2012.

[169] S. Qiao, Y. Zeng, L. Zhou, Z. Liu, and J. Ma, “A secure authentication method of intelligent terminals based on jensen-shannon divergence,' in 2017 International Conference on Networking and Network Applications (NaNA), 2017, pp. 158-163.

[170] Y. E. H. Shehadeh and D. Hogrefe, "A survey on secret key generation mechanisms on the physical layer in wireless networks," Security and Communication Networks, vol. 8, no. 2, pp. 332-341, 2015.

[171] Y. Peng, G. C. Alexandropoulos, P. Wang, Y. Li, and D. Ha, "Poster: Secret key generation from cfr for ofdm tdd systems over fading channels," in 9th International Conference on Communications and Networking in China, 2014, pp. 660-661.

[172] S. Saxena, G. Sanyal, and S. Srivastava, "Mutual authentication protocol using identity-based shared secret key in cloud environments,' in International Conference on Recent Advances and Innovations in Engineering (ICRAIE-2014), 2014, pp. 1-6.

[173] S. Sharma and A. Kaul, "A survey on intrusion detection systems and honeypot based proactive security mechanisms in vanets and vanet cloud," Vehicular communications, vol. 12, pp. 138-164, 2018.

[174] E. Illi, F. E. Bouanani, D. B. da Costa, P. C. Sofotasios, F. Ayoub, K. Mezher, and S. Muhaidat, "Physical layer security of a dual-hop regenerative mixed rf/uow system," IEEE Transactions on Sustainable Computing, vol. 6, no. 1, pp. 90-104, 2021.

[175] D. Zou, C. Gong, and Z. Xu, "Secrecy rate of miso optical wireless scattering communications," IEEE Transactions on Communications, vol. 66, no. 1, pp. 225-238, 2018.

[176] A. S. M. Badrudduza, M. Ibrahim, S. M. R. Islam, M. S. Hossen, M. K. Kundu, I. S. Ansari, and H. Yu, "Security at the physical layer over gg fading and megg turbulence induced rf-uowc mixed system," IEEE Access, vol. 9, pp. 18 123-18 136, 2021.

[177] W. K. Wootters and W. H. Zurek, "A single quantum cannot be cloned," Nature, vol. 299, no. 5886, pp. 802-803, 1982.

[178] N. Gisin, G. Ribordy, W. Tittel, and H. Zbinden, "Quantum cryptography," Rev. Mod. Phys., vol. 74, pp. 145-195, Mar 2002.

[179] H.-K. Lo, M. Curty, and K. Tamaki, "Secure quantum key distribution,"
Nature Photonics, vol. 8, no. 8, pp. 595-604, 2014.

[180] S. Pirandola, C. Ottaviani, G. Spedalieri, C. Weedbrook, S. L. Braunstein, S. Lloyd, T. Gehring, C. S. Jacobsen, and U. L. Andersen, "Highrate measurement-device-independent quantum cryptography," Nature Photonics, vol. 9, no. 6, pp. 397-402, 2015.

[181] S. L. Braunstein and P. van Loock, "Quantum information with continuous variables," Rev. Mod. Phys., vol. 77, pp. 513-577, Jun 2005.

[182] A. Leverrier, F. Grosshans, and P. Grangier, "Finite-size analysis of a continuous-variable quantum key distribution," Phys. Rev. A, vol. 81 p. 062343, Jun 2010.

[183] P. Jouguet, S. Kunz-Jacques, A. Leverrier, P. Grangier, and E. Diamanti, "Experimental demonstration of long-distance continuousvariable quantum key distribution," Nature photonics, vol. 7, no. 5, pp. 378-381, 2013

[184] T. Gehring, V. Händchen, J. Duhme, F. Furrer, T. Franz, C. Pacher, R. F. Werner, and R. Schnabel, "Implementation of continuousvariable quantum key distribution with composable and one-sideddevice-independent security against coherent attacks," Nature communications, vol. 6 , no. 1 , pp. 1-7, 2015.

[185] M. Wang, H. Wei, D. Wang, and X. Hou, "Hybrid eavesdropping approach against energy-ratio-based detection," in 2017 9th International Conference on Wireless Communications and Signal Processing (WCSP), 2017, pp. 1-6.

[186] D. Xu, P. Ren, and H. Lin, "Combat hybrid eavesdropping in powerdomain noma: Joint design of timing channel and symbol transformation," IEEE Transactions on Vehicular Technology, vol. 67, no. 6, pp. 4998-5012, 2018.

[187] J. Long, S. Xie, E. Li, C. Gao, Y. Gao, Y. Zhang, H. Zheng, G. Guo, and L. Huang, "Breakthrough the communication bottleneck between sky and underwater," AIP Advances, vol. 11, no. 2, p. 025029, 2021.

[188] S. Yan, G. Geraci, N. Yang, R. Malaney, and J. Yuan, "On the target secrecy rate for sisome wiretap channels," in 2014 IEEE International Conference on Communications (ICC), 2014, pp. 987-992.

[189] H. Yu, S. Guo, Y. Yang, and B. Xiao, "Optimal target secrecy rate and power allocation policy for a swipt system over a fading wiretap channel," IEEE Systems Journal, vol. 12, no. 4, pp. 3291-3302, 2018.

[190] Y. Zhong, X. Ge, T. Han, Q. Li, and J. Zhang, "Tradeoff between delay and physical layer security in wireless networks," IEEE Journal on Selected Areas in Communications, vol. 36, no. 7, pp. 1635-1647, 2018

[191] W. Yu, A. Chorti, L. Musavian, H. Vincent Poor, and Q. Ni, "Effective secrecy rate for a downlink noma network" IEEE Transactions on Wireless Communications, vol. 18, no. 12, pp. 5673-5690, 2019.

[192] H. Forssell, R. Thobaben, H. Al-Zubaidy, and J. Gross, "On the impact of feature-based physical layer authentication on network delay performance," in Proc. IEEE GLOBECOM, Dec. 2017, pp. 1-6.

[193] H. Forssell, R. Thobaben, H. Al-Zubaidy, and J. Gross, "Physical layer authentication in mission-critical mtc networks: A security and delay performance analysis," IEEE J. on Selected Areas in Commun., vol. 37 no. 4, pp. 795-808, Apr. 2019.

[194] W. Aman, Z. Haider, S. W. H. Shah, M. M. U. Rahman, and O. A. Dobre, "On the effective capacity of an underwater acoustic channel under impersonation attack," 2020.

[195] Y. Sun, M. Peng, Y. Zhou, Y. Huang, and S. Mao, "Application of machine learning in wireless networks: Key techniques and open issues," IEEE Communications Surveys \& Tutorials, vol. 21, no. 4, pp. 3072-3108, 2019.

[196] D. Gündüz, P. de Kerret, N. D. Sidiropoulos, D. Gesbert, C. R. Murthy, and M. van der Schaar, "Machine learning in the air," IEEE Journal on Selected Areas in Communications, vol. 37, no. 10, pp. 2184-2199, 2019.

[197] M. E. Morocho-Cayamcela, H. Lee, and W. Lim, "Machine learning for $5 \mathrm{~g} / \mathrm{b} 5 \mathrm{~g}$ mobile and wireless communications: Potential, limitations, and future directions," IEEE Access, vol. 7, pp. 137 184-137 206, 2019.

[198] G. Zhu, D. Liu, Y. Du, C. You, J. Zhang, and K. Huang, "Toward an intelligent edge: Wireless communication meets machine learning," IEEE Communications Magazine, vol. 58, no. 1, pp. 19-25, 2020.

[199] K. Sharma and M. Ghose, "Cross layer security framework for wireless sensor networks," International Journal of Security and Its Applications, vol. 5, no. 1, pp. 39-52, 2011.

[200] M. M. Farag, M. Azab, and B. Mokhtar, "Cross-layer security framework for smart grid: Physical security layer," in IEEE PES Innovative Smart Grid Technologies, Europe, 2014, pp. 1-7.

[201] J. Wurm, Y. Jin, Y. Liu, S. Hu, K. Heffner, F. Rahman, and M. Tehra- 
nipoor, "Introduction to cyber-physical system security: A cross-layer perspective," IEEE Transactions on Multi-Scale Computing Systems, vol. 3, no. 3, pp. 215-227, 2017.

[202] M. Sumalatha and V. Nandalal, "An intelligent cross layer security based fuzzy trust calculation mechanism (cls-ftcm) for securing wireless sensor network (wsn)," Journal of Ambient Intelligence and Humanized Computing, vol. 12, no. 5, pp. 4559-4573, 2021.

[203] H.-P. Tan, R. Diamant, W. K. Seah, and M. Waldmeyer, "A survey of techniques and challenges in underwater localization," Ocean Engineering, vol. 38, no. 14-15, pp. 1663-1676, 2011.

[204] I. Ullah, Y. Liu, X. Su, and P. Kim, "Efficient and accurate target localization in underwater environment," IEEE Access, vol. 7, pp. 101415-101 426, 2019.

[205] I. Ullah, J. Chen, X. Su, C. Esposito, and C. Choi, "Localization and detection of targets in underwater wireless sensor using distance and angle based algorithms," IEEE Access, vol. 7, pp. 45 693-45 704, 2019.

[206] X. Su, I. Ullah, X. Liu, and D. Choi, "A review of underwater localization techniques, algorithms, and challenges," Journal of Sensors, vol. 2020, 2020.

[207] S. Goyal, T. Bhatia, and A. Verma, "Wormhole and sybil attack in wsn: A review," in 2015 2nd International Conference on Computing for Sustainable Global Development (INDIACom). IEEE, 2015, pp. 1463-1468.
[208] M. Sharma, A. Tandon, S. Narayan, and B. Bhushan, "Classification and analysis of security attacks in wsns and ieee 802.15. 4 standards: A survey," in 2017 3rd International Conference on Advances in Computing, Communication \& Automation (ICACCA)(Fall). IEEE, 2017, pp. 1-5.

[209] P. Kaliyar, W. B. Jaballah, M. Conti, and C. Lal, "Lidl: Localization with early detection of sybil and wormhole attacks in iot networks," Computers \& Security, vol. 94, p. 101849, 2020.

[210] H. Li, Y. He, X. Cheng, H. Zhu, and L. Sun, "Security and privacy in localization for underwater sensor networks," IEEE Communications Magazine, vol. 53, no. 11, pp. 56-62, 2015.

[211] V. Baranidharan and K. Varadharajan, "Secure localization using coordinated gradient descent technique for underwater wireless sensor networks," ICTACT Journal on Communication Technology, vol. 9 , no. 1, pp. 1716-1720, 2018.

[212] M. Shanthi and D. K. Anvekar, "Secure localization for underwater wireless sensor networks based on probabilistic approach," in 2018 Second International Conference on Advances in Electronics, Computers and Communications (ICAECC). IEEE, 2018, pp. 1-6.

[213] S. Misra and T. Ojha, "Secret: Secure range-based localization with evidence theory for underwater sensor networks," ACM Transactions on Autonomous and Adaptive Systems (TAAS), vol. 15, no. 1, pp. 1-26, 2021. 